\title{
ON THE ARITHMETIC OF POWER MONOIDS AND SUMSETS IN CYCLIC GROUPS
}

\author{
AUSTIN A. ANTONIOU AND SALVATORE TRINGALI
}

\begin{abstract}
Let $H$ be a multiplicatively written monoid with identity $1_{H}$ (in particular, a group), and denote by $\mathcal{P}_{\text {fin, } \times}(H)$ the monoid obtained by endowing the collection of all finite subsets of $H$ containing a unit with the operation of setwise multiplication $(X, Y) \mapsto\{x y: x \in X, y \in Y\}$. We study fundamental features of the arithmetic of this and related structures, with a focus on the submonoid, $\mathcal{P}_{\text {fin }, 1}(H)$, of $\mathcal{P}_{\text {fin }, \times}(H)$ consisting of all finite subsets of $H$ containing the identity.

Among others, we establish that $\mathcal{P}_{\text {fin, } 1}(H)$ is atomic (i.e., each non-unit is a product of atoms) if and only if $1_{H} \neq x^{2} \neq x$ for every $x \in H \backslash\left\{1_{H}\right\}$. Then we prove that $\mathcal{P}_{\text {fin, }, 1}(H)$ is BF (i.e., it is atomic and every element has factorizations of bounded length) if and only if $H$ is torsion-free; and we show how to transfer these conclusions from $\mathcal{P}_{\text {fin }, 1}(H)$ to $\mathcal{P}_{\text {fin }, \times}(H)$ through the machinery of equimorphisms.

Next, we introduce a suitable notion of "minimal factorization" (and investigate its behavior with respect to equimorphisms) to account for the fact that monoids may have non-trivial idempotents, in which case standard definitions from Factorization Theory degenerate. Accordingly, we obtain necessary and sufficient conditions for $\mathcal{P}_{\text {fin }, \times}(H)$ to be $\mathrm{BmF}$ (meaning that each non-unit has at least one minimal factorization and all such factorizations are bounded in length); and for $\mathcal{P}_{\text {fin, } 1}(H)$ to be $\mathrm{BmF}, \mathrm{HmF}$ (i.e., a BmF-monoid where all the minimal factorizations of a given element have the same length), or minimally factorial (i.e., a BmF-monoid where each non-unit element has an essentially unique minimal factorization). Finally, we prove how to realize certain intervals as sets of minimal lengths in $\mathcal{P}_{\text {fin }, 1}(H)$.

Many proofs come down to considering sumset decompositions in cyclic groups, so giving rise to an intriguing interplay with Arithmetic Combinatorics.
\end{abstract}

\section{INTRODUCTION}

By and large, Factorization Theory is about generalizations of the Fundamental Theorem of Arithmetic from the integers to other settings: Slightly more precisely, it can be understood as the study of certain properties of rings and, more generally, monoids where factorization of elements into "irreducibles" need not be unique, see the proceedings [2,15-17], the surveys [11,12,24], or the volumes [22, 25]. While the focus has been so far on integral domains and commutative cancellative monoids, the field has recently witnessed an increasing interest for settings where cancellativity or commutativity may not be satisfied, see $[9,20,28,32,34]$ and references therein. In the present work, we further contribute to this line of research, by inquiring into the arithmetic of a new class of "highly non-cancellative" monoids recently introduced in [20] to serve as a bridge between Factorization Theory and Arithmetic Combinatorics, with emphasis on the "structural theory" of sumsets and product sets in groups [27,30,31,33].

2010 Mathematics Subject Classification. Primary 11B30, 11P70, 20M13. Secondary 11B13.

Key words and phrases. BF-monoids, decompositions into atoms, irreducibles, minimal factorizations, non-unique factorization, power monoids, product sets, sumsets.

A.A. was supported by a Rhodus Graduate Fellowship from the Department of Mathematics at the Ohio State University, and S.T. by the Austrian Science Fund (FWF), Project No. M 1900-N39. 
More in detail, let $H$ be a monoid (in particular, a group) with identity $1_{H}$ (see $\S 2$ for basic notation and terminology). The set of all non-empty finite subsets of $H$ is then also a monoid, denoted by $\mathcal{P}_{\text {fin }}(H)$ and called the power monoid of $H$, when endowed with the operation of setwise multiplication

$$
(X, Y) \mapsto X Y:=\{x y: x \in X, y \in Y\} .
$$

Moreover, the set $\mathcal{P}_{\text {fin }, \times}(H)$ of all finite subsets of $H$ containing a unit is a submonoid of $\mathcal{P}_{\text {fin }}(H)$, and so is the set $\mathcal{P}_{\text {fin }, 1}(H)$ of all finite subsets of $H$ containing $1_{H}$ : We will refer to the former as the restricted power monoid of $H$, and to the latter as the reduced power monoid of $H$.

After recalling some central ideas and objects from Factorization Theory in $\S 2$, we begin considering power monoids in $\S 3$. We explore the interplay between the restricted and the reduced power monoids, and we conclude that, under mild assumptions on $H$, their arithmetic is essentially the same. Specifically, we give necessary and sufficient conditions under which $\mathcal{P}_{\text {fin }, 1}(H)$ is atomic (Theorem 3.9); and by a combinatorial argument simply based on the Pigeonhole Principle, we conclude that $\mathcal{P}_{\text {fin }, 1}(H)$ is $\mathrm{BF}$ if and only if $H$ is torsion-free (parts (i) and (ii) of Theorem 3.11). Then we discuss how to transfer these conclusions from $\mathcal{P}_{\text {fin }, 1}(H)$ to $\mathcal{P}_{\text {fin }, \times}(H)$ through the machinery of equimorphisms (Definition 3.3), under the hypothesis that $H$ is Dedekind-finite (Proposition 3.5, Example 3.6, and Theorem 3.11(iii)).

In $\S 4$, we address a well-known limitation of Factorization Theory as developed in the classical setting: Finer arithmetic properties (e.g., boundedness of factorization lengths) are completely precluded by the existence of non-trivial idempotent elements. In the setting of power monoids this issue is nearly unavoidable (Example 3.10). We are thus motivated to introduce minimal factorizations (Definitions 4.1 and 4.4), that is, a refinement of the classical notion of factorization which, on the one hand, circumvents the problem and, on the other hand, is no different from the usual notion in the commutative, cancellative setting (Proposition 4.7(v)).

In particular, we first investigate how minimal factorizations behave with respect to equimorphisms (Propositions 4.9 and 4.10 and Example 4.11). Then we obtain necessary and sufficient conditions for $\mathcal{P}_{\text {fin }, 1}(H)$ to be BmF (meaning that the monoid is atomic and the minimal factorizations of a fixed element are all bounded in length), $\mathrm{HmF}$ (i.e., a BmF-monoid where all the minimal factorizations of a given element have the same length), or minimally factorial (i.e., a BmF-monoid where each non-unit element has an essentially unique minimal factorization); and for $\mathcal{P}_{\mathrm{fin}, \times}(H)$ to be $\mathrm{BmF}$ (Theorems 4.13 and 4.14 and Corollary 4.15). Finally in $\S 5$, we focus on $\mathcal{P}_{\text {fin }, 1}(H)$ for the special case when $H$ is a finite cyclic group or is isomorphic to the additive monoid of non-negative integers. This allows us to show (Theorem 5.7) that, for general $H$, certain intervals can be always realized as "sets of minimal lengths" in $\mathcal{P}_{\text {fin }, 1}(H)$.

\section{Preliminaries}

In this short section, we fix some definitions that we will need as we inquire into the algebraic and arithmetic structure of power monoids.

2.1. Generalities. Unless noted otherwise, we reserve the letters $\ell, m$, and $n$ (with or without subscripts) for positive integers; and the letters $i, j$, and $k$ for non-negative integers. 
We use $\mathbf{N}$ for the natural numbers (in particular, $0 \in \mathbf{N}$ ), and for $a, b \in \mathbf{R} \cup\{\infty\}$ we define $\llbracket a, b \rrbracket:=$

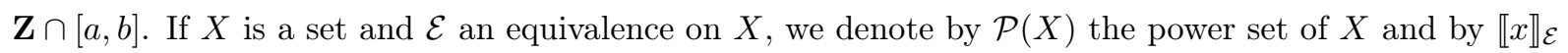
the class of an element $x \in X$ in the quotient $X / \mathcal{E}$.

When $n$ is understood from context, $\bar{k}$ will denote the residue class of $k$ modulo $n$. Occasionally, we will also need to lift residues modulo $n$ back to the integers. So, if $a \in \mathbf{Z} / n \mathbf{Z}$ and there is no risk of confusion, we set $\hat{a}:=\min (a \cap \mathbf{N}) \in \llbracket 0, n-1 \rrbracket$, and for $A \subseteq \mathbf{Z} / n \mathbf{Z}$ we define $\hat{A}:=\{\hat{a}: a \in A\}$.

Given a set $\mathscr{U}$, we denote by $\mathscr{F}(\mathscr{U})$ the free monoid with basis $\mathscr{U}$, and by $\varepsilon_{\mathscr{U}}$ the identity of $\mathscr{F}(\mathscr{U})$. We assume $\mathscr{U} \subseteq \mathscr{F}(\mathscr{U})$, and adopt the symbol $*$ for the operation of $\mathscr{F}(\mathscr{U})$. We call an element of $\mathscr{F}(\mathscr{U})$ a $\mathscr{U}$-word, and $\varepsilon_{\mathscr{U}}$ the empty $\mathscr{U}$-word. For all $\mathfrak{z} \in \mathscr{F}(\mathscr{U})$ and $n \in \mathbf{N}^{+}$, we take $\mathfrak{z}^{* 0}:=\varepsilon \mathscr{U}$, $\mathfrak{z}^{* 1}:=\mathfrak{z}$, and $\mathfrak{z}^{* n}:=\mathfrak{z}^{*(n-1)} * \mathfrak{z}$; and we define the word length, $\left\|_{\mathfrak{z}}\right\|_{\mathscr{U}}$, of $\mathfrak{z}$ (relative to the basis $\mathscr{U}$ ) as follows: If $\mathfrak{z}=\varepsilon_{\mathscr{U}}$, then $\left\|_{\mathfrak{z}}\right\|_{\mathscr{U}}:=0$; otherwise, there are (uniquely) determined $z_{1}, \ldots, z_{n} \in \mathscr{U}$ such that $\mathfrak{z}=z_{1} * \cdots * z_{n}$, and we let $\left\|_{\mathfrak{z}}\right\|_{\mathscr{U}}:=n$. Lastly, we say a $\mathscr{U}$-word $\mathfrak{y}$ is a subword of a $\mathscr{U}$-word $\mathfrak{z}$ if $\mathfrak{y}=\varepsilon \mathscr{U}$, or $\mathfrak{z}=z_{1} * \cdots * z_{n}$ and $\mathfrak{y}=z_{i_{1}} * \cdots * z_{i_{m}}$ for some $i_{1}, \ldots, i_{m} \in \llbracket 1, n \rrbracket$ with $i_{j}<i_{j+1}$ for each $j \in \llbracket 1, m-1 \rrbracket$.

Further terminology and notations, if not explained, are standard or should be clear from the context.

2.2. Basic definitions for monoids. Here and later, monoids will be usually written multiplicatively and, unless differently specified, need not have any special property (e.g., commutativity).

Let $H$ be a monoid with identity $1_{H}$. We denote by $H^{\times}$and $\mathscr{A}(H)$, respectively, the set of units (or invertible elements) and the set of atoms of $H$, where $a \in H$ is an atom if $a \notin H^{\times}$and there do not exist $x, y \in H \backslash H^{\times}$such that $a=x y$. We say that $H$ is reduced if $H^{\times}=\left\{1_{H}\right\}$; cancellative if $x z=y z$ or $z x=z y$, for some $x, y, z \in H$, implies $x=y$; Dedekind-finite if there do not exist $x, y \in H$ with $x y=1_{H} \neq y x$; and unit-cancellative provided that $x y \neq x \neq y x$ for all $x, y \in H$ with $y \notin H^{\times}$.

Given $x, y \in H$, we write $\left.x\right|_{H} y$, read " $x$ divides $y$ (in $H$ )", if $y \in H x H$; and $x \simeq_{H} y$, read " $x$ is associate to $y$ (in $H$ )", if $y \in H^{\times} x H^{\times}$. We set $\langle x\rangle_{H}:=\left\{x^{n}: n \in \mathbf{N}^{+}\right\}$and let $\operatorname{ord}_{H}(x)$ be the order of $x$ (relative to $H$ ), that is, the cardinality of $\langle x\rangle_{H}$ (when $x$ is a unit, this coincides with the common group-theoretic sense of "order"). Lastly, we call $x$ an idempotent element (of $H$ ) if $x^{2}=x$, and we take a submonoid $M$ of $H$ to be divisor-closed if $x \in M$ whenever $\left.x\right|_{H} y$ and $y \in M$.

It is perhaps worth mentioning that there are, in principle, several options for a definition of " $x$ divides $y$ " (and hence of "divisor-closedness") in a non-commutative setting, cf. [10, §5]. The definition we are choosing here is consistent with [20] and fits very well with our approach to the study of factorization in a setting that is not only non-commutative, but also non-cancellative.

2.3. Factorizations and lengths. We let the factorization homomorphism of $H$ be the unique (monoid) homomorphism $\pi_{H}: \mathscr{F}(H) \rightarrow H$ such that $\pi_{H}(x)=x$ for all $x \in H$, and we write $\mathscr{C}_{H}$ for the smallest monoid congruence on $\mathscr{F}(\mathscr{A}(H))$ for which the following holds:

- If $\mathfrak{a}=a_{1} * \cdots * a_{m}$ and $\mathfrak{b}=b_{1} * \cdots * b_{n}$ are, respectively, non-empty $\mathscr{A}(H)$-words of length $m$ and $n$, then $(\mathfrak{a}, \mathfrak{b}) \in \mathscr{C}_{H}$ if and only if $\pi_{H}(\mathfrak{a})=\pi_{H}(\mathfrak{b}), m=n$, and $a_{1} \simeq_{H} b_{\sigma(1)}, \ldots, a_{n} \simeq_{H} b_{\sigma(n)}$ for some permutation $\sigma$ of the discrete interval $\llbracket 1, n \rrbracket$.

So, if $\mathfrak{a}=a_{1} * \cdots * a_{n}$ is a non-empty $\mathscr{A}(H)$-word of length $n$ and $H$ is reduced and commutative, then

$$
\llbracket \mathfrak{a} \rrbracket_{\mathscr{C}_{H}}=\left\{a_{\sigma(1)} * \cdots * a_{\sigma(n)}: \sigma \text { is a permutation of } \llbracket 1, n \rrbracket\right\} .
$$


In addition, we define, for every $x \in H$, the set of factorizations of $x$ by

$$
\mathcal{Z}_{H}(x):=\pi_{H}^{-1}(x) \cap \mathscr{F}(\mathscr{A}(H)) ;
$$

consequently, we take

$$
\mathrm{Z}_{H}(x):=\mathcal{Z}_{H}(x) / \mathscr{C}_{H}=\left\{\llbracket \mathfrak{a} \rrbracket_{\mathscr{C}_{H}}: \mathfrak{a} \in \mathcal{Z}_{H}(x)\right\} \quad \text { and } \quad \mathrm{L}_{H}(x):=\left\{\|\mathfrak{a}\|_{H}: \mathfrak{a} \in \mathcal{Z}_{H}(x)\right\} \subseteq \mathbf{N}
$$

to be the sets of factorization classes and factorization lengths of $x$, respectively. Then we say that $H$ is

- atomic if $\mathcal{Z}_{H}(x) \neq \varnothing$ for every $x \in H \backslash H^{\times}$;

- $\mathrm{FF}$ (respectively, $\mathrm{BF}$ ) if $H$ is atomic and $\mathrm{Z}_{H}(x)$ (respectively, $\mathrm{L}_{H}(x)$ ) is finite for each $x \in H$;

- $\mathrm{HF}$ (respectively, factorial) if $\left|\mathrm{L}_{H}(x)\right|=1$ (respectively, $\left|\mathrm{Z}_{H}(x)\right|=1$ ) for all $x \in H \backslash H^{\times}$.

Of course, we have that

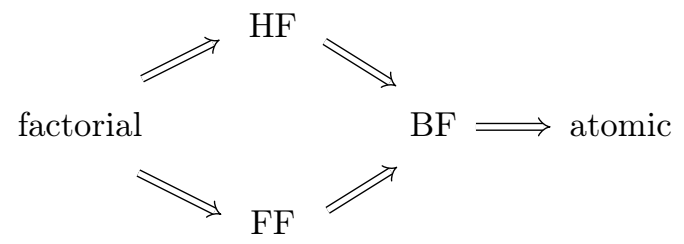

Finally, we let the system of sets of lengths of $H$ be the family

$$
\mathscr{L}(H):=\left\{\mathrm{L}_{H}(x): x \in H\right\} \backslash\{\varnothing\} \subseteq \mathcal{P}(\mathbf{N}) .
$$

2.4. Literature. Our approach to factorization in possibly non-cancellative or non-commutative monoids is borrowed from [20], where one can read thoroughly about differences and similarities with the classical approach to factorization in commutative and cancellative monoids (and hence in integral domains) pursued by A. Geroldinger and F. Halter-Koch in [25], and with the more recent approach to factorization in cancellative but possibly non-commutative monoids set forth by N. R. Baeth and D. Smertnig in [10]; in particular, see [20, Remarks 2.6 and 2.7].

This said, there are many previous entries in the literature that have treated aspects of factorization theory in commutative (unital) rings with non-trivial zero divisors. Most notably, D. D. Anderson and collaborators have extensively studied factorizations in commutative rings corresponding to notions of "associate" and "irreducible" other than the ones adopted in the present paper, see e.g. [1,3-6, 18, 19]. Below we review these alternative definitions and contrast them with our approach.

To start with, let $R$ be a commutative ring and denote by $R^{\times}$the set of units of the multiplicative monoid of $R$. Given $x, y \in R$, we say in the parlance of [5, Definition 2.1] that

- $x$ is associate to $y$ (in $R$ ), written $x \sim_{R} y$, if $x R=y R$;

- $x$ is strongly associate to $y$, written $x \approx_{R} y$, if $x \in y R^{\times}$(by Proposition 3.1(i), this is equivalent to $x$ being associate (as per $\S 2.2$ ) to $y$ in the multiplicative monoid of $R$ );

- $x$ is very strongly associate to $y$, written $x \cong_{R} y$, if $x \sim_{R} y$ and one of the following holds:

(i) $x=y=0_{R}$ (where $0_{R}$ is the zero of $R$ );

(ii) $x \neq 0_{R}$ and if $x=y z$ for some $z \in R$ then $z \in R^{\times}$.

Accordingly, one has three notions of "irreducible", see [5, Definition 2.4]. To wit, an element $a \in R$ is

- irreducible if $a \notin R^{\times}$and $a=x y$ for some $x, y \in R$ implies that $a \sim_{R} x$ or $a \sim_{R} y$;

- strongly irreducible if $a \notin R^{\times}$and $a=x y$ for some $x, y \in R$ implies that $a \approx_{R} x$ or $a \approx_{R} y$;

- very strongly irreducible if $a \notin R^{\times}$and $a=x y$ for some $x, y \in R$ implies that $a \cong_{R} x$ or $a \cong_{R} y$. 
It is obvious that very strongly irreducible elements of $R$ are strongly irreducible, and strongly irreducible elements are irreducible. In general, none of these implications can be reversed, see the paragraph after the proof of Theorem 2.12 in [5]. However, we get by [5, Theorem 2.2(3)] that the three notions coincide when $R$ is présimplifiable in the sense of [13], meaning that if $x y=x$ for some $x, y \in R$ then $x=0_{R}$ or $y \in R^{\times}$(e.g., this is the case when $R$ is an integral domain). Moreover, [5, Theorem 2.5] yields that a non-zero element of $R$ is strongly irreducible if and only if it is an atom of the multiplicative monoid of $R$.

Putting it all together, we thus see that the $\operatorname{ring} R$ is very strongly atomic in the sense of [5, Definition 3.1] if and only if one of the following holds:

(A1) $R$ has non-trivial zero divisors and the multiplicative monoid of $R$ is atomic (as per $\S 2.3$ );

(A2) $R$ is an integral domain and $R \backslash\left\{0_{R}\right\}$ is an atomic monoid under multiplication.

Similarly, $R$ is a bounded factorization ring in the sense of [5, Definition 3.8], a half-factorial ring in the sense of [7, p. 87], a finite factorization ring in the sense of [5, Definition 6.5], or a unique factorization ring in the sense of [5, Definition 4.3] if and only if one of conditions (A1) and (A2) in the above is satisfied with "atomic" replaced, respectively, by "BF", "HF", "FF", or "factorial".

As long as the scope is restricted to commutative rings, it is therefore possible to compare our approach to factorization with others based on irreducibles, strong irreducibles, or even alternative "elementary factors" (including the ones considered by C. R. Fletcher [21], A. Bouvier [13, 14], and S. Galovich [23]) by referring to $[5,18]$, where these comparisons are worked out in great detail. (Incidentally, it appears that "irreducibles" in the sense of [23] are non-units, although this is not explicitly stated by Galovich in his paper.) See also [8, Theorem 3.4 and Corollary 3.5] for a couple of results of a more arithmetic flavor concerning lengths of factorizations into irreducibles in commutative rings of the form $D / x D$, where $D$ is a principal ideal domain and $x$ is a non-zero, non-unit element of $D$.

\section{Atomicity And Bounded FACtorization in POWER MONOIDS}

Here we embark on the study of the (arithmetic and algebraic) structure of power monoids. We begin with some elementary but helpful observations we will often use without comment.

Proposition 3.1. Let $H$ be a monoid. The following hold:

(i) If $u, v \in H^{\times}$then $u v \in H^{\times}$, and the converse holds whenever $H$ is Dedekind-finite.

(ii) If $\mathscr{A}(H)$ is non-empty or $H$ is commutative or unit-cancellative, then $H$ is Dedekind-finite.

(iii) If $a \in \mathscr{A}(H)$ and $u, v \in H^{\times}$, then uav $\in \mathscr{A}(H)$.

(iv) If $x \in H \backslash H^{\times}$and $u, v \in H^{\times}$, then $\mathrm{L}_{H}(u x v)=\mathrm{L}_{H}(x)$.

Proof. See [20, parts (i), (ii), and (iv) of Lemma 2.2, and Proposition 2.30].

Proposition 3.2. Let $H$ be a monoid. The following hold:

(i) If $X_{1}, \ldots, X_{n} \in \mathcal{P}_{\text {fin }, 1}(H)$, then $X_{1} \cup \cdots \cup X_{n} \subseteq X_{1} \cdots X_{n}$.

(ii) If $u, v \in H^{\times}$and $X_{1}, \ldots, X_{n} \in \mathcal{P}_{\text {fin }, \times}(H)$, then $\left|u X_{1} \cdots X_{n} v\right|=\left|X_{1} \cdots X_{n}\right| \geq \max _{1 \leq i \leq n}\left|X_{i}\right|$.

(iii) If $K$ is a submonoid of $H$, then $\mathcal{P}_{\text {fin }, 1}(K)$ is a divisor-closed submonoid of $\mathcal{P}_{\text {fin }, 1}(H)$. (Note that the conclusion is valid regardless of whether $K$ itself is divisor-closed.)

(iv) $\mathcal{P}_{\text {fin }, 1}(H)$ is a reduced monoid and $\mathcal{P}_{\text {fin }}(H)^{\times}=\mathcal{P}_{\text {fin }, \times}(H)^{\times}=\left\{\{u\}: u \in H^{\times}\right\}$. 
(v) $\mathscr{A}\left(\mathcal{P}_{\text {fin }, \times}(H)\right) \subseteq H^{\times} \mathscr{A}\left(\mathcal{P}_{\text {fin }, 1}(H)\right) H^{\times}$.

Proof. (i) is trivial, upon considering that $\left(X \cdot 1_{H}\right) \cup\left(1_{H} \cdot Y\right) \subseteq X Y$ for all $X, Y \in \mathcal{P}_{\text {fin, }, 1}(H)$; (ii) is a direct consequence of (i) and the fact that the function $X \rightarrow H: x \mapsto u x v$ is injective for all $u, v \in H^{\times}$ and $X \subseteq H$; and (iii) and (iv) are immediate from (i) and (ii).

As for (v), let $A \in \mathscr{A}\left(\mathcal{P}_{\text {fin }, \times}(H)\right)$. Because $A$ contains a unit of $H$, there is $u \in H^{\times}$such that $1_{H} \in u A$. Then $u A$ is an element of $\mathcal{P}_{\text {fin }, 1}(H)$, and by Proposition 3.1(iii) it is also an atom of $\mathcal{P}_{\text {fin }, \times}(H)$. Thus, if $X, Y \in \mathcal{P}_{\text {fin }, 1}(H) \subseteq \mathcal{P}_{\text {fin }, \times}(H)$ and $u A=X Y$, then $X$ or $Y$ is the identity of $\mathcal{P}_{\text {fin }, 1}(H)$. This means that $u A$ is an atom of $\mathcal{P}_{\text {fin }, 1}(H)$, and hence $A=u^{-1}(u A) \in H^{\times} \mathscr{A}\left(\mathcal{P}_{\text {fin }, 1}(H)\right)$, as wished.

Our ultimate goal is, for an arbitrary monoid $H$, to investigate factorizations in $\mathcal{P}_{\text {fin }}(H)$. However, this is a difficult task in general, due to a variety of "pathological situations" that might be hard to classify in a satisfactory way, see e.g. [20, Remark 3.3(ii)].

In practice, it is more convenient to start with $\mathcal{P}_{\text {fin }, 1}(H)$ and then lift arithmetic results from $\mathcal{P}_{\text {fin }, 1}(H)$ to $\mathcal{P}_{\text {fin }, \times}(H)$, a point of view which is corroborated by the simple consideration that $\mathcal{P}_{\text {fin }}(H)=\mathcal{P}_{\text {fin }, \times}(H)$ whenever $H$ is a group (i.e., in the case of greatest interest in Arithmetic Combinatorics).

In turn, we will see that studying the arithmetic of $\mathcal{P}_{\text {fin, }, \times}(H)$ is tantamount to studying that of $\mathcal{P}_{\text {fin, }, 1}(H)$, in a sense to be made precise presently. To do so in as all-encompassing a way as possible, we recall from [34, Definition 3.2] a notion which formally packages the idea that, under suitable conditions, arithmetic may be transferred from one monoid to another.

Definition 3.3. Let $H$ and $K$ be monoids, and let $\varphi$ be a function from $H$ to $K$. We denote by $\varphi^{*}$ the unique monoid homomorphism $\mathscr{F}(H) \rightarrow \mathscr{F}(K)$ such that $\varphi^{*}(x)=\varphi(x)$ for every $x \in H$, and we call $\varphi$ an equimorphism (from $H$ to $K$ ) if $\varphi$ is a monoid homomorphism $H \rightarrow K$ and the following hold:

(E1) $\varphi^{-1}\left(K^{\times}\right) \subseteq H^{\times}$;

(E2) $\varphi$ is atom-preserving, meaning that $\varphi(\mathscr{A}(H)) \subseteq \mathscr{A}(K)$;

(E3) If $x \in H$ and $\mathfrak{b} \in \mathcal{Z}_{K}(\varphi(x))$ is a non-empty $\mathscr{A}(K)$-word, then $\varphi^{*}(\mathfrak{a}) \in \llbracket \mathfrak{b} \rrbracket_{\mathscr{C}_{K}}$ for some $\mathfrak{a} \in \mathcal{Z}_{H}(x)$. Moreover, we say that $\varphi$ is essentially surjective if $K=K^{\times} \varphi(H) K^{\times}$.

Proposition 3.4. Let $H$ and $K$ be monoids and $\varphi: H \rightarrow K$ an equimorphism. The following hold:

(i) $\mathrm{L}_{H}(x)=\mathrm{L}_{K}(\varphi(x))$ for all $x \in H \backslash H^{\times}$.

(ii) If $\varphi$ is essentially surjective, then for all $y \in K \backslash K^{\times}$there is $x \in H \backslash H^{\times}$with $\mathrm{L}_{K}(y)=\mathrm{L}_{H}(x)$.

Proof. See [20, Theorem 2.22(i)] and [34, Theorem 3.3(i)].

Proposition 3.5. Let $H$ be a Dedekind-finite monoid. The following hold:

(i) The natural embedding $\jmath: \mathcal{P}_{\mathrm{fin}, 1}(H) \hookrightarrow \mathcal{P}_{\mathrm{fin}, \times}(H)$ is an essentially surjective equimorphism.

(ii) $\mathscr{A}\left(\mathcal{P}_{\text {fin }, \times}(H)\right)=H^{\times} \mathscr{A}\left(\mathcal{P}_{\text {fin }, 1}(H)\right) H^{\times}$.

(iii) $\mathrm{L}_{\mathcal{P}_{\text {fin }, 1}(H)}(X)=\mathrm{L}_{\mathcal{P}_{\text {fin }, \times}(H)}(X)$ for every $X \in \mathcal{P}_{\text {fin }, 1}(H)$.

(iv) $\mathscr{L}\left(\mathcal{P}_{\text {fin }, \times}(H)\right)=\mathscr{L}\left(\mathcal{P}_{\text {fin }, 1}(H)\right)$.

Proof. In view of Proposition 3.4, parts (iii) and (iv) are immediate from (i). Moreover, the inclusion from left to right in (ii) is precisely the content of Proposition 3.2(v), and the other inclusion will follow from (i) and Propositions 3.1(iii) and 3.2(iv). Therefore, we focus on (i) for the remainder of the proof. 
(i) By Proposition 3.2(iv), J satisfies (E1). Moreover, $\jmath$ is essentially surjective, as any $X \in \mathcal{P}_{\text {fin, } \times}(H)$ contains a unit $u \in H^{\times}$, so $u^{-1} X \in \mathcal{P}_{\text {fin }, 1}(H)$ and $X=u\left(u^{-1} X\right)$ is associate to an element of $\mathcal{P}_{\text {fin, }, 1}(H)$.

To prove (E2), let $A \in \mathscr{A}\left(\mathcal{P}_{\text {fin, }, 1}(H)\right)$. We aim to show that $A$ is an atom of $\mathcal{P}_{\text {fin }, \times}(H)$. Suppose that $A=X Y$ for some $X, Y \in \mathcal{P}_{\text {fin }, \times}(H)$. Then there are $x \in X$ and $y \in Y$ with $x y=1_{H}$; and using that $H$ is Dedekind-finite, we get from Proposition 3.1(i) that $x, y \in H^{\times}$. It follows that

$$
A=X Y=\left(X x^{-1}\right)(x Y) \quad \text { and } \quad X x^{-1}, x Y \in \mathcal{P}_{\text {fin }, 1}(H) .
$$

But then $X x^{-1}=\left\{1_{H}\right\}$ or $x Y=\left\{1_{H}\right\}$, since $\mathcal{P}_{\text {fin }, 1}(H)$ is a reduced monoid and $A$ is an atom of $\mathcal{P}_{\text {fin, }, 1}(H)$. So, $X$ or $Y$ is a 1-element subset of $H^{\times}$, and hence $A \in \mathscr{A}\left(\mathcal{P}_{\text {fin }, \times}(H)\right)$.

It remains to show that $\mathrm{J}$ satisfies (E3). Pick $X \in \mathcal{P}_{\mathrm{fin}, 1}(H)$. If $X=\left\{1_{H}\right\}$, the conclusion holds vacuously. Otherwise, let $\mathfrak{b}:=B_{1} * \cdots * B_{n} \in \mathcal{Z}_{\mathcal{P}_{\text {fin }, 1}(H)}(X)$. Then there are $u_{1} \in B_{1}, \ldots, u_{n} \in B_{n}$ such that $1_{H}=u_{1} \cdots u_{n}$; and as in the proof of (E2), it must be that $u_{1}, \ldots, u_{n} \in H^{\times}$. Accordingly, we take, for every $i \in \llbracket 1, n \rrbracket, A_{i}:=u_{0} \cdots u_{i-1} B_{i} u_{i}^{-1} \cdots u_{1}^{-1}$, where $u_{0}:=1_{H}$. Then

$$
A_{1} \cdots A_{n}=X \quad \text { and } \quad 1_{H} \in A_{1} \cap \cdots \cap A_{n} ;
$$

and by Propositions 3.1(iii) and $3.2(\mathrm{v}), A_{1}, \ldots, A_{n}$ are atoms of $\mathcal{P}_{\text {fin, } 1}(H)$. This shows that $\mathfrak{a}:=A_{1} *$ $\cdots * A_{n} \in \mathcal{Z}_{\mathcal{P}_{\mathrm{fin}, 1}(H)}(X)$. Since $A_{i} \simeq_{\mathcal{P}_{\mathrm{fin}, \times}(H)} B_{i}$ for each $i \in \llbracket 1, n \rrbracket$ (by construction), we thus conclude that $\mathfrak{a}$ is $\mathscr{C}_{\mathcal{P}_{\text {fin }, \times}(H)}$-congruent to $\mathfrak{b}$, as wished.

The next example proves that Dedekind-finiteness is, to some extent, necessary for Proposition 3.5(ii), and hence for the subsequent conclusions.

Example 3.6. Let $\mathcal{B}$ be the set of all binary sequences $\mathfrak{s}: \mathbf{N}^{+} \rightarrow\{0,1\}$, and let $H$ denote the monoid of all functions $\mathcal{B} \rightarrow \mathcal{B}$ under composition. We will write $H$ multiplicatively; so, if $f, g \in H$ then $f g$ is the map $\mathcal{B} \rightarrow \mathcal{B}: \mathfrak{s} \mapsto f(g(\mathfrak{s}))$. Further, let $n \geq 5$ and consider the functions

$$
\begin{aligned}
& L: \mathcal{B} \rightarrow \mathcal{B}:\left(a_{1}, a_{2}, \ldots\right) \mapsto\left(a_{2}, a_{3}, \ldots\right) \\
& \text { (left shift); } \\
& R: \mathcal{B} \rightarrow \mathcal{B}:\left(a_{1}, a_{2}, \ldots\right) \mapsto\left(0, a_{1}, a_{2}, \ldots\right) \\
& \text { (right shift); } \\
& P: \mathcal{B} \rightarrow \mathcal{B}:\left(a_{1}, a_{2}, \ldots\right) \mapsto\left(a_{n}, a_{1}, \ldots, a_{n-1}, a_{n+1}, a_{n+2}, \ldots\right)
\end{aligned}
$$

In particular, $P \in H^{\times}$. Also, $L R=\mathrm{id}_{\mathcal{B}}$ but $R L \neq \mathrm{id}_{\mathcal{B}}$; whence $H$ is not Dedekind-finite, and neither $R$ nor $L$ is invertible. With this in mind, we will prove that $A:=\{L, P\} \cdot\{R, P\}=\left\{\operatorname{id}_{\mathcal{B}}, L P, P R, P^{2}\right\}$ is an atom of $\mathcal{P}_{\text {fin, } 1}(H)$, although it is not, by construction, an atom of $\mathcal{P}_{\text {fin, } \times}(H)$.

Indeed, assume $A=X Y$ for some $X, Y \in \mathcal{P}_{\text {fin }, 1}(H)$. Then $X, Y \subseteq A$, and it is clear that $P^{2} \neq P R L P$, or else $R L=\operatorname{id}_{\mathcal{B}}$ (a contradiction). Similarly, $P R P R \neq P^{2} \neq L P L P$; otherwise, $P=R P R$ and hence $R$ is invertible, or $P=L P L$ and $L$ is invertible (again a contradiction). Lastly, we see that $P^{2} \neq L P^{2} R$, by applying both $P^{2}$ and $L P^{2} R$ to the constant sequence $(1,1, \ldots)$.

It follows that $P^{2}$ must belong to $X$ or $Y$, but not to both (which is the reason for choosing $n \geq 5$ ). Accordingly, let $P^{2} \in X \backslash Y$ (the other case is analogous). Then $Y=\left\{\operatorname{id}_{\mathcal{B}}\right\}$, since one can easily check that $P^{2} L P, P^{3} R \notin A$, by noting that the action of $P^{2} L P$ and $P^{3} R$ differ from that of $A$ on the sequences $(1,1, \ldots)$ and $(1,0,1,1, \ldots)$. This makes $A$ an atom of $\mathcal{P}_{\text {fin }, 1}(H)$.

We get from Proposition 3.5 that studying factorization properties of $\mathcal{P}_{\text {fin }, 1}(H)$ is sufficient for studying corresponding properties of $\mathcal{P}_{\text {fin }, \times}(H)$, at least in the case when $H$ is Dedekind-finite. Thus, as a 
starting point in the investigation of the arithmetic of $\mathcal{P}_{\mathrm{fin}, 1}(H)$, one might wish to give a comprehensive description of the atoms of $\mathcal{P}_{\text {fin, },}(H)$. This is however an overwhelming task even in specific cases (e.g., when $H$ is the additive group of the integers), let alone the general case. Nevertheless, we can obtain basic information about $\mathscr{A}\left(\mathcal{P}_{\text {fin }, 1}(H)\right)$ in full generality.

Lemma 3.7. Let $H$ be a monoid and $x \in H \backslash\left\{1_{H}\right\}$. The following hold:

(i) The set $\left\{1_{H}, x\right\}$ is an atom of $\mathcal{P}_{\mathrm{fin}, 1}(H)$ if and only if $1_{H} \neq x^{2} \neq x$.

(ii) If $x^{2}=1_{H}$ or $x^{2}=x$, then $\left\{1_{H}, x\right\}$ factors into a product of atoms neither in $\mathcal{P}_{\text {fin, }, 1}(H)$ nor in $\mathcal{P}_{\text {fin }, \times}(H)$.

Proof. (i) If $x^{2}=1_{H}$ or $x^{2}=x$, then it is clear that $\left\{1_{H}, x\right\}=\left\{1_{H}, x\right\}^{2}$, and therefore $\left\{1_{H}, x\right\}$ is not an atom of $\mathcal{P}_{\text {fin }, 1}(H)$. As for the converse, assume that $\left\{1_{H}, x\right\}=Y Z$ for some non-units $Y, Z \in \mathcal{P}_{\text {fin }, 1}(H)$. Then we get from Proposition 3.2 that $Y$ and $Z$ are 2-element sets, namely, $Y=\left\{1_{H}, y\right\}$ and $Z=\left\{1_{H}, z\right\}$ with $y, z \in H \backslash\left\{1_{H}\right\}$. Hence $\left\{1_{H}, x\right\}=Y Z=\left\{1_{H}, y, z, y z\right\}$, and immediately this implies $x=y=z$. Therefore, $\left\{1_{H}, x\right\}=\left\{1_{H}, x, x^{2}\right\}$, which is only possible if $x^{2}=1_{H}$ or $x^{2}=x$.

(ii) Suppose that $x^{2}=1_{H}$ or $x^{2}=x$. Then the calculation above shows that $\left\{1_{H}, x\right\}=\left\{1_{H}, x\right\}^{2}$ and there is no other decomposition of $\left\{1_{H}, x\right\}$ into a product of non-unit elements of $\mathcal{P}_{\text {fin, }, 1}(H)$. So, $\left\{1_{H}, x\right\}$ is a non-trivial idempotent (hence, a non-unit) and has no factorization into atoms of $\mathcal{P}_{\text {fin, }, 1}(H)$.

It remains to prove the analogous statement for $\mathcal{P}_{\text {fin }, \times}(H)$. Assume to the contrary that $\left\{1_{H}, x\right\}$ factors into a product of $n$ atoms of $\mathcal{P}_{\text {fin }, \times}(H)$ for some $n \in \mathbf{N}^{+}$. Then $n \geq 2$, since $\left\{1_{H}, x\right\}$ is a non-trivial idempotent (and hence not an atom itself). Consequently, we can write $\left\{1_{H}, x\right\}=Y Z$, where $Y$ is an atom and $Z$ a non-unit of $\mathcal{P}_{\mathrm{fin}, \times}(H)$. In particular, we get from parts (i), (ii), and (iv) of Proposition 3.2 that both $Y$ and $Z$ are 2-element sets, say, $Y=\{u, y\}$ and $Z=\{v, z\}$. It is then immediate that there are only two possibilities: $1_{H}$ is the product of two units from $Y$ and $Z$, or the product of two non-units from $Y$ and $Z$. Without loss of generality, we are thus reduced to considering the following cases.

CASE 1: $u v=1_{H}$. Then $u z \neq 1_{H}$ (or else $z=u^{-1}=v$, contradicting the fact that $Z$ is a 2-element set). So $u z=x$, and similarly $y v=x$. Then $y=x u=u z u$ and $z=x v=v y v$, and therefore

$$
\{u, y\}=\{u, u z u\}=\left\{1_{H}, u z\right\}\{u\}=\left\{1_{H}, x\right\}\{u\}=\{u, y\}\{v u, z u\} .
$$

However, this shows that $\{u, y\}$ is not an atom of $\mathcal{P}_{\text {fin, } \times}(H)$, in contrast with our assumptions.

CASE 2: $y z=1_{H}$ and $y, z \in H \backslash H^{\times}$. Then $u, v \in H^{\times}$, by the fact that $\{u, y\},\{v, z\} \in \mathcal{P}_{\text {fin }, \times}(H)$; and we must have $u z=x$, for $u z=1_{H}$ would yield $z=u^{-1} \in H^{\times}$. In particular, $x=u z$ is not a unit in $H$, so $u v=1_{H}$ and we are back to the previous case.

We have just seen that, to even hope for $\mathcal{P}_{\text {fin }, 1}(H)$ to be atomic, we need that the "bottom layer" of 2-element subsets of $H$ consists only of atoms; perhaps surprisingly, it will turn out that such a condition is also sufficient. Before proving this, we point out some structural implications of the fact that every non-identity element of $H$ is neither an idempotent nor a square root of $1_{H}$.

Lemma 3.8. Let $H$ be a monoid such that $1_{H} \neq x^{2} \neq x$ for all $x \in H \backslash\left\{1_{H}\right\}$. The following hold:

(i) $H$ is Dedekind-finite.

(ii) If $x \in H \backslash\left\{1_{H}\right\}$ and $\langle x\rangle_{H}$ is finite, then $x \in H^{\times}$and $\langle x\rangle_{H}$ is a cyclic group of order $\geq 3$. 
Proof. (i) Let $y, z \in H$ such that $y z=1_{H}$. Then $(z y)^{2}=z(y z) y=z y$, and since $H$ has no non-trivial idempotents, we conclude that $z y=1_{H}$. Consequently, $H$ is Dedekind-finite.

(ii) This is an obvious consequence of [35, Ch. V, Exercise 4, p. 68], according to which every finite semigroup has an idempotent. The proof is short, so we give it here for the sake of self-containedness.

Because $\langle x\rangle_{H}$ is finite, there exist $n, k \in \mathbf{N}^{+}$such that $x^{n}=x^{n+k}$, and by induction this implies that $x^{n}=x^{n+h k}$ for all $h \in \mathbf{N}$. Therefore, we find that

$$
\left(x^{n k}\right)^{2}=x^{2 n k}=x^{(k+1) n} x^{(k-1) n}=x^{n} x^{(k-1) n}=x^{n k} .
$$

But $H$ has no non-trivial idempotents, thus it must be the case that $x^{n k}=1_{H}$. That is, $x$ is a unit of $H$, and we have $x^{-1}=x^{n k-1} \in\langle x\rangle_{H}$. So, $\langle x\rangle_{H}$ is a (finite) cyclic group of order $\geq 3$.

Theorem 3.9. Let $H$ be a monoid. Then $\mathcal{P}_{\mathrm{fin}, 1}(H)$ is atomic if and only if $1_{H} \neq x^{2} \neq x$ for every $x \in H \backslash\left\{1_{H}\right\}$.

Proof. The "only if" part is a consequence of Lemma 3.7(ii). As for the other direction, assume that $1_{H} \neq x^{2} \neq x$ for each $x \in H \backslash\left\{1_{H}\right\}$, and fix $X \in \mathcal{P}_{\text {fin }, 1}(H)$ with $|X| \geq 2$. We wish to show that

$$
X=A_{1} \cdots A_{n}, \quad \text { for some } A_{1}, \ldots, A_{n} \in \mathscr{A}\left(\mathcal{P}_{\text {fin }, 1}(H)\right) .
$$

If $X$ is a 2-element set, the claim is true by Lemma 3.7(i). So let $|X| \geq 3$, and suppose inductively that every $Y \in \mathcal{P}_{\text {fin, }, 1}(H)$ with $2 \leq|Y|<|X|$ is a product of atoms. If $X$ is an atom, we are done. Otherwise, $X=A B$ for some non-units $A, B \in \mathcal{P}_{\text {fin }, 1}(H)$, and by symmetry we can assume $|X| \geq|A| \geq|B| \geq 2$.

If $|A|<|X|$, then both $A$ and $B$ factor into a product of atoms (by the inductive hypothesis), and so too does $X=A B$. Consequently, we are only left to consider the case when $|X|=|A|$.

For, we notice that $A \cup B \subseteq A B=X$ (because $1_{H} \in A \cap B$ ), and this is only possible if $A=X$ (since $|A|=|X|$ and $A \subseteq X)$. So, to summarize, we have that

$$
|X| \geq 3, \quad|B| \geq 2, \quad \text { and } \quad B \subseteq A B=X=A .
$$

In particular, since $B$ is not a unit of $\mathcal{P}_{\text {fin }, 1}(H)$, we can choose an element $b \in B \backslash\left\{1_{H}\right\} \subseteq A$. Hence, taking $A_{b}:=A \backslash\{b\}$, we have $\left|A_{b}\right|<|A|$, and it is easy to check that $A_{b} B=A=X$ (in fact, $1_{H}$ is in $A_{b} \cap B$, and therefore we derive from (1) that $A_{b} B \subseteq A=A_{b} \cup\{b\} \subseteq A_{b} B \cup\{b\} \subseteq A_{b} B \cup B=A_{b} B$ ).

If $|B|<|A|$, then we are done, because $A_{b}$ and $B$ are both products of atoms (by the inductive hypothesis), and thus so is $X=A B=A_{b} B$. Otherwise, it follows from (1) and the above that

$$
X=A=B=A_{b} B \quad \text { and } \quad|A| \geq 3,
$$

so we can choose an element $a \in A \backslash\left\{1_{H}, b\right\}$. Accordingly, set $B_{a}:=B \backslash\{a\}$. Then $\left|B_{a}\right|<|B|$ (because $A=B$ and $a \in A$ ), and both $A_{b}$ and $B_{a}$ decompose into a product of atoms (again by induction). But this finishes the proof, since it is straightforward from (2) that $X=A=A_{b} B_{a}$ (indeed, $1_{H} \in A_{b} \cap B_{a}$ and $b \in B_{a}$, so we find that $A_{b} B_{b} \subseteq A=A_{b} \cup\{b\} \subseteq A_{b} B_{a} \cup\{b\} \subseteq A_{b} B_{a} \cup B_{a}=A_{b} B_{a}$ ).

Now with Proposition 3.4 and Theorem 3.9 in hand, we can engage in a finer study of the arithmetic of power monoids; in particular, we may wish to study their (systems of) sets of lengths. However, we are immediately met with a "problem" (i.e., some sets of lengths are infinite in a rather trivial way): 
Example 3.10. Let $H$ be a monoid with an element $x$ of finite odd order $m \geq 3$, and set $X:=\left\{x^{k}: k \in\right.$ $\mathbf{N}\}$. Then it is clear that $X$ is the setwise product of $n$ copies of $\left\{1_{H}, x\right\}$ for every $n \geq m$. This shows that the set of lengths of $X$ relative to $\mathcal{P}_{\text {fin, } 1}(H)$ contains $\llbracket m, \infty \rrbracket$ (and hence is infinite), since we know from Lemma 3.7 that $\left\{1_{H}, x\right\}$ is an atom of $\mathcal{P}_{\text {fin, }, 1}(H)$.

The nature of this problem is better clarified by our next result, and we will more thoroughly address it in $\S 4$.

Theorem 3.11. Let $H$ be a monoid. The following hold:

(i) If $H$ is torsion-free and $X \in \mathcal{P}_{\text {fin }, 1}(H)$, then $\sup \left\llcorner_{\mathcal{P}_{\text {fin }, 1}(H)}(X) \leq|X|^{2}-|X|\right.$.

(ii) $\mathcal{P}_{\text {fin }, 1}(H)$ is $\mathrm{BF}$ if and only if $H$ is torsion-free.

(iii) $\mathcal{P}_{\text {fin }, \times}(H)$ is $\mathrm{BF}$ if and only if so is $\mathcal{P}_{\text {fin }, 1}(H)$.

Proof. (i) Set $n:=|X| \in \mathbf{N}^{+}$, fix an integer $\ell \geq(n-1) n+1$, and suppose for a contradiction that $X=$ $A_{1} \cdots A_{\ell}$ for some $A_{1}, \ldots, A_{\ell} \in \mathscr{A}\left(\mathcal{P}_{\text {fin }, 1}(H)\right)$. By the Pigeonhole Principle, there are an element $x \in X$ and a subset $I \subseteq \llbracket 1, \ell \rrbracket$ such that $m:=|I| \geq n$ and $x \in A_{i}$ for each $i \in I$. So, writing $I=\left\{i_{1}, \ldots, i_{m}\right\}$, we find that $x^{k} \in A_{i_{1}} \cdots A_{i_{k}} \subseteq A_{1} \cdots A_{\ell}=X$ for every $k \in \llbracket 1, m \rrbracket$, i.e., $\left\{1_{H}, x, \ldots, x^{m}\right\} \subseteq X$. However, since $H$ is torsion-free, each power of $x$ is distinct, and hence $n=|X| \geq m+1>n$ (a contradiction).

(ii) First suppose for a contradiction that $\mathcal{P}_{\text {fin }, 1}(H)$ is $\mathrm{BF}$ and has an element $x$ of finite order $m$. Then $\mathcal{P}_{\text {fin, }, 1}(H)$ is also atomic, and we know by Theorem 3.9 and Lemma 3.8(ii) that $x^{m}=1_{H}$. If $m$ is even, then $\left(x^{m / 2}\right)^{2}=1_{H}$, contradicting the atomicity of $\mathcal{P}_{\text {fin }, 1}(H)$ since, by Theorem 3.9, no non-identity element of $H$ can have order 2. If $m$ is odd, then Example 3.10 shows that the set of lengths of $\left\{x^{k}: k \in \mathbf{N}\right\}$ is infinite, contradicting the assumption that $\mathcal{P}_{\text {fin }, 1}(H)$ is $\mathrm{BF}$.

Conversely, assume $H$ is torsion-free. Then all powers of non-identity elements are distinct, so Theorem 3.9 implies that $\mathcal{P}_{\text {fin, }, 1}(H)$ is atomic, and (i) gives an explicit upper bound on the lengths of factorizations.

(iii) The "only if" part follows from [20, Theorem 2.28(iv) and Corollary 2.29], so suppose that $\mathcal{P}_{\text {fin }, 1}(H)$ is BF. Then $\mathcal{P}_{\text {fin, }, 1}(H)$ is atomic, and hence, by Theorem $3.9,1_{H} \neq x^{2} \neq x$ for all $x \in H \backslash\left\{1_{H}\right\}$. By Lemma 3.8(i), this implies that $H$ is Dedekind-finite, so the natural embedding $\mathcal{P}_{\text {fin }, 1}(H) \hookrightarrow \mathcal{P}_{\text {fin }, \times}(H)$ is an essentially surjective equimorphism by Proposition 3.5(i). The result is then an immediate consequence of Proposition 3.5(iv).

\section{Minimal faCtorizations and COnditions For Bounded minimal Lengths}

Example 3.10 indicates that, in the presence of torsion in the ground monoid $H$, sets of lengths in $\mathcal{P}_{\text {fin }, 1}(H)$ blow up in a predictable fashion, with the result that most of the invariants classically studied in Factorization Theory lose their significance. In the case of Example 3.10, this phenomenon is due to the existence of non-trivial idempotents and has been previously addressed by many authors in the literature on commutative rings and monoids (see Remarks 4.5 and 4.6). Here we strive for a "natural approach" that applies to arbitrary monoids, spurring us to consider a refinement of the notions introduced in $\S 2.3$ and to investigate some of their fundamental properties (see, in particular, Definition 4.4 and Proposition 4.9 ), before focusing on the special case of power monoids.

4.1. Minimal factorizations. We start with the definition of a binary relation (in fact, a preorder) on the $\mathscr{A}(H)$-words of a monoid $H$ that we shall use to "filter out the redundant factors" that may contribute 
to the factorizations of an element of $H$ (recall that, given a set $X$, we denote by $\mathscr{F}(X)$ the free monoid with basis $X$ and by $\varepsilon_{X}$ the identity of $\left.\mathscr{F}(X)\right)$.

Definition 4.1. Let $H$ be a monoid. We denote by $\preceq_{H}$ the binary relation on $\mathscr{F}(\mathscr{A}(H))$ determined by taking $\mathfrak{a} \preceq_{H} \mathfrak{b}$, for some $\mathscr{A}(H)$-words $\mathfrak{a}$ and $\mathfrak{b}$, if and only if either $\mathfrak{a}=\mathfrak{b}=\varepsilon_{\mathscr{A}(H)}$; or $\mathfrak{a}$ and $\mathfrak{b}$ are non-empty words of length $m$ and $n$ respectively, say $\mathfrak{a}=a_{1} * \cdots * a_{m}$ and $\mathfrak{b}=b_{1} * \cdots * b_{n}$, such that $\pi_{H}(\mathfrak{a})=\pi_{H}(\mathfrak{b})$ and $a_{\sigma(1)} \simeq_{H} b_{1}, \ldots, a_{\sigma(m)} \simeq_{H} b_{m}$ for some injection $\sigma: \llbracket 1, m \rrbracket \rightarrow \llbracket 1, n \rrbracket$.

We say an $\mathscr{A}(H)$-word $\mathfrak{a}$ is $\preceq_{H}$-minimal, or simply minimal (when no confusion can arise), if there is no $\mathscr{A}(H)$-word $\mathfrak{b}$ with $\mathfrak{b} \prec_{H} \mathfrak{a}$, where $\mathfrak{b} \prec_{H} \mathfrak{a}$ means that $\mathfrak{b} \preceq_{H} \mathfrak{a}$ but $\mathfrak{a} \swarrow_{H} \mathfrak{b}$.

Remark 4.2. The preorder $\preceq_{H}$ in Definition 4.1 is closely related to the "divides-up-to-permutation" relation $\left.\right|_{p}$ considered by N.R. Baeth and D. Smertnig in [10, Definition 5.2(2)], when the latter is specialized to the free monoid $\mathscr{F}(\mathscr{A}(H))$ : More precisely, $\mathfrak{a} \preceq \mathfrak{b}$ if and only if $\left.\mathfrak{a}\right|_{p} \mathfrak{b}$ and $\pi_{H}(\mathfrak{a})=\pi_{H}(\mathfrak{b})$.

The next result highlights a few basic properties of the relation introduced in Definition 4.1.

Proposition 4.3. Let $H$ be a monoid, and let $\mathfrak{a}, \mathfrak{b} \in \mathscr{F}(\mathscr{A}(H))$. The following hold:

(i) $\preceq_{H}$ is a preorder (i.e., a reflexive and transitive binary relation) on $\mathscr{F}(\mathscr{A}(H))$.

(ii) If $\mathfrak{a} \preceq_{H} \mathfrak{b}$ then $\|\mathfrak{a}\|_{H} \leq\|\mathfrak{b}\|_{H}$.

(iii) $\mathfrak{a} \preceq_{H} \mathfrak{b}$ and $\mathfrak{b} \preceq_{H} \mathfrak{a}$ if and only if $\mathfrak{a} \preceq_{H} \mathfrak{b}$ and $\|\mathfrak{a}\|_{H}=\|\mathfrak{b}\|_{H}$, if and only if $(\mathfrak{a}, \mathfrak{b}) \in \mathscr{C}_{H}$.

Proof. Points (i) and (ii) are straightforward from our definitions.

As for (iii), set $h:=\|\mathfrak{a}\|_{H}$ and $k:=\|\mathfrak{b}\|_{H}$. By part (ii), $\mathfrak{a} \preceq_{H} \mathfrak{b}$ and $\mathfrak{b} \preceq_{H} \mathfrak{a}$ only if $\mathfrak{a} \preceq_{H} \mathfrak{b}$ and $h=k$; and it is immediate to check that $(\mathfrak{a}, \mathfrak{b}) \in \mathscr{C}_{H}$ implies $\mathfrak{a} \preceq_{H} \mathfrak{b}$ and $\mathfrak{b} \preceq_{H} \mathfrak{a}$. So, to finish the proof, assume that $\mathfrak{a} \preceq_{H} \mathfrak{b}$ and $h=k$. We only need to show that $(\mathfrak{a}, \mathfrak{b}) \in \mathscr{C}_{H}$. For, we have (by definition) that $\mathfrak{a} \preceq_{H} \mathfrak{b}$ if and only if $\pi_{H}(\mathfrak{a})=\pi_{H}(\mathfrak{b})$ and there is an injection $\sigma: \llbracket 1, h \rrbracket \rightarrow \llbracket 1, k \rrbracket$ such that $a_{i} \simeq_{H} b_{\sigma(i)}$ for every $i \in \llbracket 1, h \rrbracket$. But $\sigma$ is actually a bijection (because $h=k$ ), and we can thus conclude that $(\mathfrak{a}, \mathfrak{b}) \in \mathscr{C}_{H}$.

Definition 4.4. Let $H$ be a monoid, and let $x \in H$. We refer to a $\preceq_{H}$-minimal $\mathscr{A}(H)$-word $\mathfrak{a}$ such that $x=\pi_{H}(\mathfrak{a})$ as a $\preceq_{H}$-minimal factorization of $x$, or simply as a minimal factorization of $x$ provided $H$ is clear from context. Accordingly, we denote by

$$
\mathcal{Z}_{H}^{\mathrm{m}}(x):=\left\{\mathfrak{a} \in \mathcal{Z}_{H}(x): \mathfrak{a} \text { is } \preceq H_{\text {-minimal }}\right\} \quad \text { and } \quad Z_{H}^{\mathrm{m}}(x):=\mathcal{Z}_{H}^{\mathrm{m}}(x) / \mathscr{C}_{H}
$$

the set of $\preceq_{H}$-minimal factorizations and the set of $\preceq_{H}$-minimal factorization classes of $x$, respectively (cf. the definitions from $\S 2.3$ ). In addition, we take

$$
\mathrm{L}_{H}^{\mathrm{m}}(x):=\left\{\|\mathfrak{a}\|_{H}: \mathfrak{a} \in \mathcal{Z}_{H}^{\mathrm{m}}(x)\right\} \subseteq \mathbf{N}
$$

to be the set of $\preceq_{H}$-minimal factorization lengths of $x$, and

$$
\mathscr{L}^{\mathrm{m}}(H):=\left\{\mathrm{L}_{H}^{\mathrm{m}}(x): x \in H\right\} \subseteq \mathcal{P}(\mathbf{N})
$$

to be the system of sets of $\preceq_{H}$-minimal lengths of $H$. Lastly, we say that the monoid $H$ is

- $\mathrm{BmF}$ or bounded-minimally-factorial (respectively, FmF or finite-minimally-factorial) if $\mathrm{L}_{H}^{\mathrm{m}}(x)$ (respectively, $\mathrm{Z}_{H}^{\mathrm{m}}(x)$ ) is finite and non-empty for every $x \in H \backslash H^{\times}$;

- $\mathrm{HmF}$ or half-minimally-factorial (respectively, minimally factorial) if $\mathrm{L}_{H}^{\mathrm{m}}(x)$ (respectively, $\left.\mathrm{Z}_{H}^{\mathrm{m}}(x)\right)$ is a singleton for all $x \in H \backslash H^{\times}$. 
Note that we may write $\mathcal{Z}^{\mathrm{m}}(x)$ for $\mathcal{Z}_{H}^{\mathrm{m}}(x), \mathrm{L}^{\mathrm{m}}(x)$ for $\mathrm{L}_{H}^{\mathrm{m}}(x)$, etc. if there is no likelihood of confusion.

Remark 4.5. To the best of our knowledge, analogues of the notions introduced in Definition 4.4 have only been considered so far in a commutative setting, with one significant example being offered by the work of S. Chun, D. D. Anderson, and S. Valdes-Leon [19] on "reduced factorizations".

In detail, let $H$ be the multiplicative monoid of a (unital) ring $R$ and fix a set $\mathcal{A} \subseteq R$. We say that a non-empty $\mathcal{A}$-word $a_{1} * \cdots * a_{n}$ of length $n$ is a minimal $\mathcal{A}$-factorization of an element $x \in R$ if $\pi_{H}(\mathfrak{a})=x$ but $x \neq \pi_{H}(\mathfrak{b})$ for every non-empty $\mathcal{A}$-word $\mathfrak{b}=b_{1} * \cdots * b_{m}$ of length $m \leq n-1$ for which there exists an injection $\sigma: \llbracket 1, m \rrbracket \rightarrow \llbracket 1, n \rrbracket$ such that $b_{i} \simeq_{H} a_{\sigma(i)}$ for each $i \in \llbracket 1, m \rrbracket$.

A minimal $\mathscr{A}(H)$-factorization of a non-unit $x \in R$ is the same as a $\preceq_{H}$-minimal factorization of $x$ (as per Definition 4.4). Moreover, it follows from $\S 2.4$ and Proposition 3.1(iv) that, if $R$ is a commutative ring and $x$ is not the zero of $R$, then a minimal $\mathscr{A}(H)$-factorization of $x$ is, in the parlance of [19, Definition 2.1 and $\S 3$, essentially the same as a strongly $\mu$-reduced $\mu$-factorization of $x$ into very strongly irreducible elements of $R$. Insofar as the discussion is restricted to commutative rings, one can thus refer to [19] and [7] for a comparison of our approach to the study of "minimal factorizations" with others in the literature, including the one by C. R. Fletcher [21] and generalizations thereof where the set $\mathcal{A}$ in the above consists of various types of "irreducible elements" of $R$ (cf. $\S 2.4$ ).

Remark 4.6. Another approach for managing the "excess factorizations" arising from the presence of torsion (though still in a commutative setting), was outlined by A. Geroldinger and G. Lettl in [26].

In short, let $H$ be a commutative monoid and denote by $\mathcal{A}$ the set of all $a \in H \backslash H^{\times}$such that $\left.b\right|_{H} a$ only if $b \in H^{\times}$or $a H=b H$. Given $u \in H$, we define

$$
\operatorname{ind}_{H}^{\mathrm{GL}}(u):=\inf \left\{r \in \mathbf{N}: u^{i} H=u^{j} H \text { for all } i, j \geq r\right\} .
$$

Accordingly, we take a GL-factorization of a non-unit $x \in H$ to be a non-empty $\mathcal{A}$-word $\mathfrak{a}=a_{1} * \cdots * a_{n}$ such that $\pi_{H}(\mathfrak{a})=x$ and $\mathrm{v}_{a}^{H}(\mathfrak{a}) \leq \operatorname{ind}_{H}^{\mathrm{GL}}(a)$ for every $a \in \mathcal{A}$, where

$$
\mathrm{v}_{a}^{H}(\mathfrak{a}):=\left|\left\{i \in \llbracket 1, n \rrbracket: a_{i}=a\right\}\right| .
$$

A GL-factorization is fundamentally the same as the "canonical form" of a factorization in the sense of [26]; and since it is easily checked that $\mathscr{A}(H) \subseteq \mathcal{A}$, every $\preceq_{H}$-minimal factorization is also a GL-factorization. Moreover, the two notions coincide on the level of commutative, unit-cancellative monoids, in which case $\mathcal{A}=\mathscr{A}(H)$ and $\operatorname{ind}_{H}^{\mathrm{GL}}(u)=\infty$ for every non-unit $u \in H$. However, big differences exist in general. E.g., it follows from Lemma 3.7 (i) and the above that $\{\overline{0}, \overline{1}\} *\{\overline{0}, \overline{2}\} *\{\overline{0}, \overline{3}\} *\{\overline{0}, \overline{4}\}$ is a GL-factorization of $\mathbf{Z} / 5 \mathbf{Z}$ in the reduced power monoid of the cyclic group $(\mathbf{Z} / 5 \mathbf{Z},+)$; but is not a minimal factorization as per Definition 4.4 , because $\mathbf{Z} / 5 \mathbf{Z}=\{\overline{0}, \overline{1}\}+\{\overline{0}, \overline{2}\}+\{\overline{0}, \overline{3}\}$.

It is helpful, at this juncture, to observe some fundamental features of minimal factorizations.

Proposition 4.7. Let $H$ be a monoid and let $x \in H$. The following hold:

(i) Any $\mathscr{A}(H)$-word of length 0,1 , or 2 is minimal.

(ii) $\mathcal{Z}_{H}(x) \neq \varnothing$ if and only if $\mathcal{Z}_{H}^{\mathrm{m}}(x) \neq \varnothing$.

(iii) If $\mathfrak{a} \in \mathcal{Z}_{H}^{\mathrm{m}}(x)$ and $(\mathfrak{a}, \mathfrak{b}) \in \mathscr{C}_{H}$, then $\mathfrak{b} \in \mathcal{Z}_{H}^{\mathrm{m}}(x)$.

(iv) If $K$ is a divisor-closed submonoid of $H$ and $x \in K$, then $\mathcal{Z}_{K}^{\mathrm{m}}(x)=\mathcal{Z}_{H}^{\mathrm{m}}(x)$ and $\mathrm{L}_{K}^{\mathrm{m}}(x)=\mathrm{L}_{H}^{\mathrm{m}}(x)$.

(v) If $H$ is commutative and unit-cancellative, then $\mathcal{Z}_{H}^{\mathrm{m}}(x)=\mathcal{Z}_{H}(x)$, and hence $\mathrm{L}_{H}^{\mathrm{m}}(x)=\mathrm{L}_{H}(x)$. 
Proof. (i), (ii), and (iii) are an immediate consequence of parts (ii)-(iii) of Proposition 4.3 (in particular, note that, if $\mathfrak{a}$ is an $\mathscr{A}(H)$-word of length 1 , then $\pi_{H}(\mathfrak{a})$ is an atom of $H$, and therefore $\pi_{H}(\mathfrak{a}) \neq \pi_{H}(\mathfrak{b})$ for every $\mathscr{A}(H)$-words $\mathfrak{b}$ of length $\geq 2$ ); and (iv) follows at once from considering that, if $K$ is a divisor-closed submonoid of $H$ and $x \in K$, then $\mathcal{Z}_{K}(x)=\mathcal{Z}_{H}(x)$ and $\mathrm{L}_{K}(x)=\mathrm{L}_{H}(x)$, see [20, Proposition 2.21(ii)].

(v) Assume $H$ is commutative and unit-cancellative. It suffices to check that no non-empty $\mathscr{A}(H)$-word $\mathfrak{a}$ has a proper subword $\mathfrak{b}$ with $\pi_{H}(\mathfrak{a}) \simeq_{H} \pi_{H}(\mathfrak{b})$. Suppose to the contrary that there exist $a_{1}, \ldots, a_{n} \in$ $\mathscr{A}(H)$ with $\prod_{i \in I} a_{i} \simeq_{H} a_{1} \cdots a_{n}$ for some $I \subsetneq \llbracket 1, n \rrbracket$. Since $H$ is commutative, we can assume without loss of generality that $I=\llbracket 1, k \rrbracket$ for some $k \in \llbracket 0, n-1 \rrbracket$. Then unit-cancellativity implies $a_{k+1} \cdots a_{n} \in H^{\times}$, and we get from parts (i) and (ii) of Proposition 3.1 that $a_{k+1}, \ldots, a_{n} \in H^{\times}$, which is however impossible (by definition of an atom).

To further elucidate the behavior of minimal factorizations, we give an analogue of Proposition 3.1(iv) showing that multiplying a non-unit by units does not change its set of minimal factorizations.

Lemma 4.8. Let $H$ be a monoid, and fix $x \in H \backslash H^{\times}$and $u, v \in H^{\times}$. Then there is a length-preserving bijection $\mathcal{Z}_{H}^{\mathrm{m}}(x) \rightarrow \mathcal{Z}_{H}^{\mathrm{m}}(u x v)$, and in particular $\mathrm{L}_{H}^{\mathrm{m}}(x)=\mathrm{L}_{H}^{\mathrm{m}}(u x v)$.

Proof. Given $w, z \in H$ and a non-empty word $\mathfrak{z}=y_{1} * \cdots * y_{n} \in \mathscr{F}(H)$ of length $n$, denote by $w \mathfrak{z} z$ the length- $n$ word $\bar{y}_{1} * \cdots * \bar{y}_{n} \in \mathscr{F}(H)$ defined by taking $\bar{y}_{1}:=w y_{1} z$ if $n=1$, and $\bar{y}_{1}:=w y_{1}, \bar{y}_{n}:=y_{n} z$, and $\bar{y}_{i}:=y_{i}$ for all $i \in \llbracket 2, n-1 \rrbracket$ otherwise. We claim that the function

$$
f: \mathcal{Z}_{H}^{\mathrm{m}}(x) \rightarrow \mathcal{Z}_{H}^{\mathrm{m}}(u x v): \mathfrak{a} \mapsto u \mathfrak{a} v
$$

is a well-defined length-preserving bijection. In fact, it is sufficient to show that $f$ is well-defined, since this will in turn imply that the map $g: \mathcal{Z}_{H}^{\mathrm{m}}(u x v) \rightarrow \mathcal{Z}_{H}^{\mathrm{m}}(x): \mathfrak{b} \mapsto u^{-1} \mathfrak{b} v^{-1}$ is also well-defined (observe that $u x v \in H \backslash H^{\times}$and $\left.x=u^{-1} u x v v^{-1}\right)$, and then it is easy to check that $g$ is the inverse of $f$.

For the claim, let $\mathfrak{a} \in \mathcal{Z}_{H}^{\mathrm{m}}(x)$, and note that, by parts (i) and (ii) of Proposition 3.1, $\|\mathfrak{a}\|_{H}$ is a positive integer, so that $\mathfrak{a}=a_{1} * \cdots * a_{n}$ for some $a_{1}, \ldots, a_{n} \in \mathscr{A}(H)$. In view of Proposition 3.1(iii), $u \mathfrak{a} v$ is a factorization of $u x v$, and we only need to verify that it is also $\preceq_{H}$-minimal. For, suppose to the contrary that $\mathfrak{b} \prec_{H} u \mathfrak{a} v$ for some $\mathfrak{b} \in \mathscr{F}(\mathscr{A}(H))$. Then $\pi_{H}(\mathfrak{b})=\pi_{H}(u \mathfrak{a} v)=u x v$ and, by Proposition 4.3(iii), $k:=\|\mathfrak{b}\|_{H} \in \llbracket 1, n-1 \rrbracket$ (recall that $u x v \notin H^{\times}$). So, $\mathfrak{b}=b_{1} * \cdots * b_{k}$ for some atoms $b_{1}, \ldots, b_{k} \in H$, and there exists an injection $\sigma: \llbracket 1, k \rrbracket \rightarrow \llbracket 1, n \rrbracket$ such that $b_{i} \simeq_{H} a_{\sigma(i)}$ for each $i \in \llbracket 1, k \rrbracket$. Define $\mathfrak{c}:=u^{-1} \mathfrak{b} v^{-1}$.

By construction and Proposition 3.1(iii), there are $c_{1}, \ldots, c_{k} \in \mathscr{A}(H)$ such that $\mathfrak{c}=c_{1} * \cdots * c_{k}$; and it follows from the above that $\pi_{H}(\mathfrak{c})=u^{-1} \pi_{H}(\mathfrak{b}) v^{-1}=x$ and $c_{i} \simeq_{H} a_{\sigma(i)}$ for every $i \in \llbracket 1, k \rrbracket$. Since $k<n$, we can thus conclude from Proposition 4.3 (iii) that $\mathfrak{c} \prec_{H} \mathfrak{a}$, contradicting the $\preceq_{H}$-minimality of $\mathfrak{a}$.

We saw in the previous section that equimorphisms transfer factorizations between monoids (Proposition 3.4). Equimorphisms have a similar compatibility with minimal factorizations, in the sense that an equimorphism also satisfies a "minimal version" of condition (E3) from Definition 3.3.

Proposition 4.9. Let $H$ and $K$ be monoids and $\varphi: H \rightarrow K$ an equimorphism. The following hold:

(i) If $x \in H \backslash H^{\times}$and $\mathfrak{b} \in \mathcal{Z}_{K}^{\mathrm{m}}(\varphi(x))$, then there is $\mathfrak{a} \in \mathcal{Z}_{H}^{\mathrm{m}}(x)$ with $\varphi^{*}(\mathfrak{a}) \in \llbracket \mathfrak{b} \rrbracket_{\mathscr{C}_{K}}$.

(ii) $\mathrm{L}_{K}^{\mathrm{m}}(\varphi(x)) \subseteq \mathrm{L}_{H}^{\mathrm{m}}(x)$ for every $x \in H \backslash H^{\times}$.

(iii) If $\varphi$ is essentially surjective then, for all $y \in K \backslash K^{\times}$, there is $x \in H \backslash H^{\times}$with $\mathrm{L}_{K}^{\mathrm{m}}(y) \subseteq \mathrm{L}_{H}^{\mathrm{m}}(x)$. 
Proof. (i) Pick $x \in H \backslash H^{\times}$, and let $\mathfrak{b} \in \mathcal{Z}_{K}^{\mathrm{m}}(\varphi(x))$. Then $\mathfrak{b} \neq \varepsilon_{\mathscr{A}(K)}$, otherwise $\varphi(x)=\pi_{K}(\mathfrak{b})=1_{K}$ and, by (E1), $x \in \varphi^{-1}(\varphi(x))=\varphi^{-1}\left(1_{K}\right) \subseteq H^{\times}$(a contradiction). Consequently, (E3) yields the existence of a factorization $\mathfrak{a} \in \mathcal{Z}_{H}(x)$ with $\varphi^{*}(\mathfrak{a}) \in \llbracket \mathfrak{b} \rrbracket_{\mathscr{C}_{K}}$, and it only remains to show that $\mathfrak{a}$ is $\preceq_{H}$-minimal.

Note that $n:=\|\mathfrak{a}\|_{H}=\left\|\varphi^{*}(\mathfrak{a})\right\|_{K}=\|\mathfrak{b}\|_{K} \geq 1$, and write $\mathfrak{a}=a_{1} * \cdots * a_{n}$ and $\mathfrak{b}=b_{1} * \cdots * b_{n}$, with $a_{1}, \ldots, a_{n} \in \mathscr{A}(H)$ and $b_{1}, \ldots, b_{n} \in \mathscr{A}(K)$. Then suppose to the contrary that $\mathfrak{a}$ is not $\preceq_{H}$-minimal, i.e., there exist a (necessarily non-empty) $\mathscr{A}(H)$-word $\mathfrak{c}=c_{1} * \cdots * c_{m}$ and an injection $\sigma: \llbracket 1, m \rrbracket \rightarrow \llbracket 1, n \rrbracket$ such that $\pi_{H}(\mathfrak{c})=\pi_{H}(\mathfrak{a})=x$ and $c_{i} \simeq_{H} a_{\sigma(i)}$ for every $i \in \llbracket 1, m \rrbracket$. Then

$$
\pi_{K}\left(\varphi^{*}(\mathfrak{c})\right)=\varphi\left(c_{1}\right) \cdots \varphi\left(c_{m}\right)=\varphi(x) \quad \text { and } \quad \varphi\left(c_{1}\right) \simeq_{K} \varphi\left(a_{\sigma(1)}\right), \ldots, \varphi\left(c_{m}\right) \simeq_{K} \varphi\left(a_{\sigma(m)}\right)
$$

(recall that monoid homomorphisms map units to units; so, if $u \simeq_{H} v$, then $\varphi(u) \simeq_{K} \varphi(v)$ ); and together with Proposition 4.7(iii), this proves that $\varphi^{*}(\mathfrak{c}) \prec_{K} \mathfrak{b}$, contradicting the $\preceq_{K}$-minimality of $\mathfrak{b}$.

(ii) Fix $x \in H \backslash H^{\times}$, and suppose $\mathrm{L}_{K}^{\mathrm{m}}(\varphi(x)) \neq \varnothing$ (otherwise there is nothing to prove). Accordingly, let $k \in \mathrm{L}_{K}^{\mathrm{m}}(\varphi(x))$ and $\mathfrak{b} \in \mathcal{Z}_{K}^{\mathrm{m}}(\varphi(x))$ such that $k=\|\mathfrak{b}\|_{K}$. It is sufficient to check that $k \in \mathrm{L}_{H}^{\mathrm{m}}(x)$, and this is straightforward: Indeed, we have by (i) that $\varphi^{*}(\mathfrak{a})$ is $\mathscr{C}_{K}$-congruent to $\mathfrak{b}$ for some $\mathfrak{a} \in \mathcal{Z}_{H}^{\mathfrak{m}}(x)$, which implies in particular that $k=\left\|\varphi^{*}(\mathfrak{a})\right\|_{K}=\|\mathfrak{a}\|_{H} \in \mathrm{L}_{H}^{\mathrm{m}}(x)$.

(iii) Assume $\varphi$ is essentially surjective, and let $y \in K \backslash K^{\times}$. Then $y=u \varphi(x) v$ for some $u, v \in K^{\times}$ and $x \in H$, and neither $x$ is a unit of $H$ nor $\varphi(x)$ is a unit of $K$ (because $\varphi\left(H^{\times}\right) \subseteq K^{\times}$and $y \notin K^{\times}$). Accordingly, we have by Lemma 4.8 and part (ii) that $\mathrm{L}_{K}^{\mathrm{m}}(y)=\mathrm{L}_{K}^{\mathrm{m}}(\varphi(x)) \subseteq \mathrm{L}_{H}^{\mathrm{m}}(x)$.

4.2. Minimal factorizations in power monoids. Let $H$ be a monoid. Similarly as in $\S 3$, we would like to simplify the study of minimal factorizations in $\mathcal{P}_{\text {fin }, \times}(H)$ as much as possible by passing to consideration of the reduced monoid $\mathcal{P}_{\text {fin }, 1}(H)$. For, it is of primary importance to make clear the nature of the relationship between minimal factorizations in $\mathcal{P}_{\text {fin }, \times}(H)$ and those in $\mathcal{P}_{\text {fin }, 1}(H)$. We shall see that this is possible under some circumstances.

Proposition 4.10. Let $H$ be a commutative monoid, and let $X \in \mathcal{P}_{\text {fin }, 1}(H)$. The following hold:

(i) $\mathcal{Z}_{\mathcal{P}_{\text {fin }, 1}(H)}(X) \subseteq \mathcal{Z}_{\mathcal{P}_{\text {fin }, \times}(H)}(X)$.

(ii) $\mathrm{L}_{\mathcal{P}_{\text {fin }, 1}(H)}^{\mathrm{m}}(X)=\mathrm{L}_{\mathcal{P}_{\text {fin }, \times}(H)}^{\mathrm{m}}(X)$.

(iii) $\mathscr{L}^{\mathrm{m}}\left(\mathcal{P}_{\text {fin }, 1}(H)\right)=\mathscr{L}^{\mathrm{m}}\left(\mathcal{P}_{\text {fin }, \times}(H)\right)$.

Proof. (i) Let $\mathfrak{a}$ be a minimal factorization of $X$ relative to $\mathcal{P}_{\text {fin }, 1}(H)$. In light of Proposition 4.7(i), $\mathfrak{a}$ is a non-empty $\mathscr{A}\left(\mathcal{P}_{\text {fin }, 1}(H)\right)$-word, i.e., $\mathfrak{a}=A_{1} * \cdots * A_{n}$ for some atoms $A_{1}, \ldots, A_{n} \in \mathcal{P}_{\text {fin }, 1}(H)$.

Assume for the sake of contradiction that $\mathfrak{a}$ is not a minimal factorization relative to $\mathcal{P}_{\text {fin }, \times}(H)$. Then there exist a non-empty $\mathscr{A}\left(\mathcal{P}_{\text {fin }, \times}(H)\right)$-word $\mathfrak{b}=B_{1} * \cdots * B_{m}$ and an injection $\sigma: \llbracket 1, m \rrbracket \rightarrow \llbracket 1, n \rrbracket$ with

$$
X=A_{1} \cdots A_{n}=B_{1} \cdots B_{m} \quad \text { and } \quad B_{1} \simeq_{\mathcal{P}_{\text {fin }, \times}(H)} A_{\sigma(1)}, \ldots, B_{m} \simeq_{\mathcal{P}_{\text {fin }, \times}(H)} A_{\sigma(m)},
$$

and on account of Proposition 4.3(iii) we must have $1 \leq m<n$. Since $H$ is a commutative monoid, this means in particular that, for each $i \in \llbracket 1, m \rrbracket$, there is $u_{i} \in H^{\times}$such that $B_{i}=u_{i} A_{\sigma(i)}$. Thus we have

$$
A_{1} \cdots A_{n}=B_{1} \cdots B_{m}=\left(u_{1} A_{\sigma(1)}\right) \cdots\left(u_{m} A_{\sigma(m)}\right)=u \cdot A_{\sigma(1)} \cdots A_{\sigma(m)},
$$

where $u:=u_{1} \cdots u_{m} \in H^{\times}$. In view of Proposition 3.2(ii), it follows that

$$
\left|A_{1} \cdots A_{n}\right|=\left|A_{\sigma(1)} \cdots A_{\sigma(m)}\right|,
$$


which is only possible if

$$
X=A_{1} \cdots A_{n}=A_{\sigma(1)} \cdots A_{\sigma(m)},
$$

because $1_{H} \in A_{i}$ for every $i \in \llbracket 1, n \rrbracket$, and hence $A_{\sigma(1)} \cdots A_{\sigma(m)} \subseteq A_{1} \cdots A_{n}$ (note that here we use again that $H$ is commutative). So, letting $\mathfrak{a}^{\prime}$ be the $\mathscr{A}\left(\mathcal{P}_{\mathrm{fin}, 1}(H)\right)$-word $A_{\sigma(1)} * \cdots * A_{\sigma(m)}$ and recalling from the above that $m \leq n-1$, we see by Proposition 4.3 (iii) that $\mathfrak{a}^{\prime} \prec_{\mathcal{P}_{\mathrm{fin}, 1}(H)} \mathfrak{a}$, which contradicts the hypothesis that $\mathfrak{a}$ is a minimal factorization of $X$ in $\mathcal{P}_{\text {fin, }, 1}(H)$.

(ii) It is an immediate consequence of part (i) and Propositions 3.5(i) and 4.9(ii), when considering that every commutative monoid is Dedekind-finite.

(iii) We already know from part (ii) that $\mathscr{L}^{\mathrm{m}}\left(\mathcal{P}_{\text {fin }, 1}(H)\right) \subseteq \mathscr{L}^{\mathrm{m}}\left(\mathcal{P}_{\text {fin }, \times}(H)\right)$. For the opposite inclusion, fix $X \in \mathcal{P}_{\text {fin }, \times}(H)$. We claim that there exists $Y \in \mathcal{P}_{\text {fin }, 1}(H)$ with $\mathrm{L}_{\mathcal{P}_{\text {fin }, \times}(H)}(X)=\mathrm{L}_{\mathcal{P}_{\text {fin }, 1}(H)}(Y)$. Indeed, pick $x \in X \cap H^{\times}$. Then $x^{-1} X \in \mathcal{P}_{\text {fin,1 }}(H)$, and we derive from Lemma 4.8 and part (ii) that

$$
\mathrm{L}_{\mathcal{P}_{\text {fin }, \times}(H)}^{m}(X)=\mathrm{L}_{\mathcal{P}_{\text {fin }, \times}(H)}^{m}\left(x^{-1} X\right)=\mathrm{L}_{\mathcal{P}_{\text {fin }, 1}(H)}^{m}\left(x^{-1} X\right)
$$

which proves our claim and suffices to finish the proof (since $X$ was arbitrary).

We will now discuss an instance in which equality in Proposition 4.10(ii) does not necessarily hold true in the absence of commutativity, and the best we can hope for is the containment relation implied by Proposition 4.9(ii) when $\varphi$ is the natural embedding of Proposition 3.5(i).

Example 4.11. Let $n$ be a (positive) multiple of 105, and $p$ a (positive) prime dividing $n^{2}+n+1$; note that $p \geq 11$ and $3 \leq n \bmod p \leq p-3($ where $n \bmod p$ is the remainder of the Euclidean division of $n$ by $p$ ). Following [29, p. 27], we take $H$ to be the metacyclic group generated by the 2-element set $\{r, s\}$ subject to $\operatorname{ord}_{H}(r)=p, \operatorname{ord}_{H}(s)=3$, and $s^{-1} r s=r^{n}$. Then $H$ is a non-abelian group of odd order $3 p$, and by Theorem 3.9 and Propositions 3.4(ii) and 3.5(i), $\mathcal{P}_{\text {fin }, 1}(H)$ and $\mathcal{P}_{\text {fin }, \times}(H)$ are atomic monoids.

We claim that $X:=\langle r\rangle_{H}$ has minimal factorizations of length $p-1$ in $\mathcal{P}_{\text {fin }, 1}(H)$ but not in $\mathcal{P}_{\text {fin, } \times}(H)$. Pick $g \in X \backslash\left\{1_{H}\right\}$. Clearly $\operatorname{ord}_{H}(g)=p$, and thus we get from Lemma 3.7(i) that $\left\{1_{H}, g\right\}$ is an atom of $\mathcal{P}_{\text {fin }, 1}(H)$. Then it is immediate to see that $\mathfrak{a}_{g}:=\left\{1_{H}, g\right\}^{*(p-1)}$ is a minimal factorization of $X$ in $\mathcal{P}_{\text {fin, }, 1}(H)$; most notably, $\mathfrak{a}_{g}$ is minimal since otherwise there should exist an exponent $k \in \llbracket 1, p-2 \rrbracket$ such that $g^{p-1}=g^{k}$, contradicting that $\operatorname{ord}_{H}(g)=p$. Yet, $\mathfrak{a}_{g}$ is not a minimal factorization of $X$ in $\mathcal{P}_{\text {fin, } \times}(H)$. Indeed, Proposition 3.5(ii) and Lemma 3.7(i) guarantee that $\left\{1_{H}, g\right\}$ and $\left\{1_{H}, g^{n}\right\}$ are associate atoms of $\mathcal{P}_{\text {fin }, \times}(H)$, because $s^{-1} g^{n} s=g$ and, hence, $s^{-1}\{1, g\} s=\left\{1_{H}, g^{n}\right\}$. So, in view of Proposition 4.3(iii), it is straightforward that

$$
\left\{1_{H}, g\right\}^{*(p-2)} *\left\{1_{H}, g^{n}\right\} \prec_{\mathcal{P}_{\text {fin }, \times}(H)} \mathfrak{a}_{g},
$$

In particular, note here that we have used that $3 \leq n \bmod p \leq p-3$ to obtain

$$
\left\{1_{H}, g, \ldots, g^{p-2}\right\} \cup\left\{g^{n}, g^{n+1}, \ldots, g^{n+p-2}\right\}=\left\{1_{H}, g, \ldots, g^{p-1}\right\}=X
$$

Given that, suppose for a contradiction that $X$ has a minimal factorization $\mathfrak{c}$ of length $p-1$ in $\mathcal{P}_{\text {fin, } \times}(H)$. Then by Propositions $3.5(\mathrm{i})$ and $4.9(\mathrm{i}), \mathfrak{c}$ is $\mathscr{C}_{\mathcal{P}_{\mathrm{fin}, \times}(H)^{- \text {-congruent }}}$ to a $\preceq_{\mathcal{P}_{\mathrm{fin}, 1}(H)}$-minimal factorization $\mathfrak{a}=A_{1} * \cdots * A_{p-1}$ of $X$ of length $p-1$; and we aim to show that $\mathfrak{a}$ is $\mathscr{C}_{\mathcal{P}_{\mathrm{fin}, 1}(H)}$-congruent to $\mathfrak{a}_{g}$ for some $g \in X \backslash\left\{1_{H}\right\}$, which is however impossible as it would mean that $\mathfrak{a}_{g}$ is a minimal factorization of $X$ in $\mathcal{P}_{\text {fin }, \times}(H)$, in contradiction to what established in the above. 
Indeed, let $B_{i}$ be, for $i \in \llbracket 1, p-1 \rrbracket$, the image of $\left\{k \in \llbracket 0, p-1 \rrbracket: r^{k} \in A_{i}\right\} \subseteq \mathbf{Z}$ under the canonical map $\mathbf{Z} \rightarrow \mathbf{Z} / p \mathbf{Z}$. Then $\mathfrak{a}$ is a minimal factorization of $X$ in $\mathcal{P}_{\text {fin, }, 1}(H)$ only if $\mathfrak{b}:=B_{1} * \cdots * B_{p-1}$ is a minimal factorization of $\mathbf{Z} / p \mathbf{Z}$ in the reduced power monoid of $(\mathbf{Z} / p \mathbf{Z},+)$, herein denoted by $\mathcal{P}_{\text {fin }, 0}(\mathbf{Z} / p \mathbf{Z})$.

We want to show that $\mathfrak{b}$ is $\preceq_{\mathcal{P}_{\text {fin }, 0}(H)}$-minimal only if there is a non-zero $x \in \mathbf{Z} / p \mathbf{Z}$ such that $B_{i}=\{\overline{0}, x\}$ or $B_{i}=\{\overline{0},-x\}$, or equivalently $A_{i}=\left\{1_{H}, r^{\hat{x}}\right\}$ or $A_{i}=\left\{1_{H}, r^{-\hat{x}}\right\}$, for every $i \in \llbracket 1, p-1 \rrbracket$ (for notation, see $\S 2.1$. By the preceding arguments, this will suffice to conclude that $p-1 \notin \mathrm{L}_{\mathcal{P}_{\mathrm{fin}, \times}(H)}^{\mathrm{m}}(X)$, because it implies at once that $\mathfrak{a}$ is $\mathcal{C}_{\mathcal{P}_{\mathrm{fin}, 1}(H)}$-congruent to $\mathfrak{a}_{g}$ with $g:=r^{\hat{x}} \in X \backslash\left\{1_{H}\right\}$.

To begin, let $K$ be a subset of $\llbracket 1, p-1 \rrbracket$, and define $\mathcal{S}_{K}:=\sum_{k \in K} B_{k}$ and $s_{K}:=\left\{k \in K:\left|B_{k}\right| \geq 3\right\}$. Then we have by the Cauchy-Davenport inequality (see, e.g., [30, Theorem 6.2]) that

$$
\mathcal{S}_{K}=\mathbf{Z} / p \mathbf{Z} \quad \text { or } \quad\left|\mathcal{S}_{K}\right| \geq 1+\sum_{k \in K}\left(\left|B_{k}\right|-1\right) \geq 1+|K|+s_{K} .
$$

Now, let $I$ and $J$ be disjoint subsets of $\llbracket 1, p-1 \rrbracket$ with $|I \cup J|=|I|+|J|=p-2$. We claim $s_{I}=s_{J}=0$. Indeed, it is clear that $\mathcal{S}_{I \cup J} \neq \mathbf{Z} / p \mathbf{Z}$, otherwise $\mathfrak{b}$ would not be a minimal factorization in $\mathcal{P}_{\text {fin, }, 0}(\mathbf{Z} / p \mathbf{Z})$. So, another application of the Cauchy-Davenport inequality, combined with (3), yields

$$
\left|S_{I \cup J}\right|=\left|S_{I}+S_{J}\right| \geq\left|S_{I}\right|+\left|S_{J}\right|-1 \geq 1+|I|+|J|+s_{I}+s_{J}=p-1+s_{I}+s_{J} .
$$

This suffices to prove that $\left|S_{I}+S_{J}\right|=p-1$ and $s_{I}=s_{J}=0$, or else $S_{I \cup J}=\mathbf{Z} / p \mathbf{Z}$ (a contradiction).

It follows $\left|B_{1}\right|=\cdots=\left|B_{p-1}\right|=2$. So, taking $I$ in (4) to range over all 1-element subsets of $\llbracket 1, p-1 \rrbracket$ and observing that, consequently, $\left|S_{J}\right| \geq p-1-\left|S_{I}\right|=p-3 \geq 8>\left|S_{I}\right|$, we infer from Vosper's theorem (see, e.g., [30, Theorem 8.1]) that there exists a non-zero $x \in \mathbf{Z} / p \mathbf{Z}$ such that, for every $i \in \llbracket 1, p-1 \rrbracket, B_{i}$ is an arithmetic progression of $\mathbf{Z} / p \mathbf{Z}$ with difference $x$, i.e., $B_{i}=\{\overline{0}, x\}$ or $B_{i}=\{\overline{0},-x\}$ (as wished).

We proceed with an analogue of Theorem 3.11(i) and then prove the main results of the section.

Proposition 4.12. Let $H$ be a monoid and $X \in \mathcal{P}_{\text {fin }, \times}(H)$. The following hold:

(i) If $X \in \mathcal{P}_{\text {fin, }, 1}(H)$, then a minimal factorization of $X$ in $\mathcal{P}_{\text {fin }, 1}(H)$ has length $\leq|X|-1$.

(ii) If $H$ is Dedekind-finite, then a minimal factorization of $X$ in $\mathcal{P}_{\text {fin }, \times}(H)$ has length $\leq|X|-1$.

Proof. (i) The claim is trivial if $X=\left\{1_{H}\right\}$, when the only factorization of $X$ is the empty word; or if $X \in \mathscr{A}\left(\mathcal{P}_{\text {fin }, 1}(H)\right)$, in which case $|X| \geq 2$ and $X$ has a unique factorization (of length 1 ). So, assume that $X$ is neither the identity nor an atom of $\mathcal{P}_{\text {fin, }, 1}(H)$, and let $\mathfrak{a}$ be a minimal factorization of $X$ (relative to $\left.\mathcal{P}_{\text {fin }, 1}(H)\right)$. Then $\mathfrak{a}=A_{1} * \cdots * A_{n}$, where $A_{1}, \ldots, A_{n} \in \mathscr{A}\left(\mathcal{P}_{\text {fin }, 1}(H)\right)$ and $n \geq 2$; and we claim that

$$
A_{1} \cdots A_{i} \subsetneq A_{1} \cdots A_{i+1}, \quad \text { for every } i \in \llbracket 1, n-1 \rrbracket .
$$

In fact, let $i \in \llbracket 1, n-1 \rrbracket$. Since $1_{H} \in A_{i+1}$, it is clear that $A_{1} \cdots A_{i} \subsetneq A_{1} \cdots A_{i+1}$; and the inclusion must be strict, or else $A_{1} * \cdots * A_{i} * \mathfrak{b} \prec_{\mathcal{P}_{\text {fin }, 1}(H)} \mathfrak{a}$, where $\mathfrak{b}:=\varepsilon_{\mathscr{A}\left(\mathcal{P}_{\mathrm{fin}, 1}(H)\right)}$ if $i=n-1$ and $\mathfrak{b}:=A_{i+2} * \cdots * A_{n}$ otherwise (contradicting the minimality of $\mathfrak{a}$ ). Consequently, we see that $2 \leq\left|A_{1} \cdots A_{i}\right|<\left|A_{1} \cdots A_{i+1}\right| \leq$ $|X|$ for all $i \in \llbracket 1, n-1 \rrbracket$, and this implies at once that $n \leq|X|-1$.

(ii) The conclusion is immediate from part (i) and Propositions 3.5(i) and 4.9(iii).

Theorem 4.13. Let $H$ be a monoid. Then the following are equivalent:

(a) $1_{H} \neq x^{2} \neq x$ for every $x \in H \backslash\left\{1_{H}\right\}$.

(b) $\mathcal{P}_{\text {fin }, 1}(H)$ is atomic.

(c) $\mathcal{P}_{\text {fin }, 1}(H)$ is $\mathrm{BmF}$. 
(d) $\mathcal{P}_{\text {fin }, 1}(H)$ is FmF.

(e) Every 2-element subset $X$ of $H$ with $1_{H} \in X$ is an atom of $\mathcal{P}_{\text {fin, } 1}(H)$.

(f) $\mathcal{P}_{\text {fin }, \times}(H)$ is atomic.

(g) $\mathcal{P}_{\text {fin }, \times}(H)$ is $\mathrm{BmF}$.

(h) $\mathcal{P}_{\text {fin }, \times}(H)$ is $\mathrm{FmF}$.

(i) Every 2-element subset $X$ of $H$ with $X \cap H^{\times} \neq \varnothing$ is an atom of $\mathcal{P}_{\text {fin }, \times}(H)$.

Proof. We already know from Theorem 3.9 and Lemma 3.7 that (b) $\Leftrightarrow$ (a) $\Leftrightarrow$ (e) and (i) $\Rightarrow$ (a); while it is straightforward from our definitions that $(\mathrm{h}) \Rightarrow(\mathrm{g}) \Rightarrow(\mathrm{f})$. So, it will suffice to prove that (b) $\Rightarrow(\mathrm{c})$ $\Rightarrow(\mathrm{d}) \Rightarrow(\mathrm{h})$ and $(\mathrm{f}) \Rightarrow(\mathrm{i})$.

(b) $\Rightarrow$ (c): If $X \in \mathcal{P}_{\text {fin }, 1}(H)$ is a non-unit, then $\mathcal{Z}_{\mathcal{P}_{\text {fin }, 1}(H)}(X)$ is non-empty, and by Propositions 4.7 (ii) and $4.12(\mathrm{i})$ we have that $\varnothing \neq \mathrm{L}_{\mathcal{P}_{\text {fin }, 1}(H)}^{\mathrm{m}}(X) \subseteq \llbracket 1,|X|-1 \rrbracket$. So, $\mathcal{P}_{\text {fin }, 1}(H)$ is $\mathrm{BmF}$.

$(\mathrm{c}) \Rightarrow(\mathrm{d})$ : Let $X \in \mathcal{P}_{\mathrm{fin}, 1}(H)$ be a non-unit. By Proposition 3.2(i), any atom of $\mathcal{P}_{\text {fin }, 1}(H) \operatorname{dividing} X$ must be a subset of $X$, and there are only finitely many of these (since $X$ is finite). Because a minimal factorization of $X$ is a bounded $\mathscr{A}\left(\mathcal{P}_{\text {fin, }, 1}(H)\right.$ )-word (by the assumption that $H$ is $\mathrm{BmF}$ ), it follows that $X$ has finitely many minimal factorizations, and hence $\mathcal{P}_{\text {fin }, 1}(H)$ is $\operatorname{FmF}$ (since $X$ was arbitrary).

$(\mathrm{d}) \Rightarrow(\mathrm{h})$ : Pick a non-unit $X \in \mathcal{P}_{\text {fin }, \times}(H)$, and let $u \in H^{\times}$such that $u X \in \mathcal{P}_{\text {fin }, 1}(H)$. Since $\mathcal{P}_{\text {fin }, 1}(H)$ is $\mathrm{FmF}$ (by hypothesis), it is also atomic. Hence, by Theorem 3.9 and Lemma 3.8(i), $H$ is Dedekind-finite, and so we have by Proposition 3.5(i) that the natural embedding $\mathcal{P}_{\text {fin }, 1}(H) \hookrightarrow \mathcal{P}_{\text {fin, } \times}(H)$ is an essentially surjective equimorphism. In particular, we infer from Proposition 4.9(i) that any minimal factorization

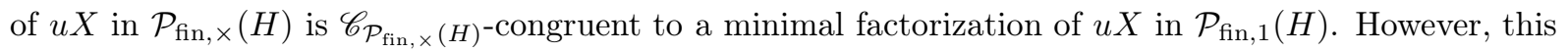
makes $Z_{\mathcal{P}_{\text {fin }, \times}(H)}^{m}(u X)$ finite, whence $Z_{\mathcal{P}_{\text {fin }, \times}(H)}^{m}(X)$ must also be finite as a consequence of Lemma 4.8 .

(f) $\Rightarrow$ (i): Let $X$ be a 2-element subset of $H$ with $X \cap H^{\times} \neq \varnothing$. Then $X=u A$ for some unit $u \in H^{\times}$, where $A:=u^{-1} X$ is a 2-element subset of $H$ with $1_{H} \in H$; and since $\mathcal{P}_{\text {fin }, \times}(H)$ is atomic (by hypothesis), we are guaranteed by Lemmas 3.7 and $3.8(\mathrm{i})$ that $A$ is an atom of $\mathcal{P}_{\text {fin, }, 1}(H)$ and $H$ is Dedekind-finite. Therefore, we conclude from Proposition 3.5(ii) that $X \in \mathscr{A}\left(\mathcal{P}_{\text {fin }, \times}(H)\right)$.

Theorem 4.14. Let $H$ be a monoid. Then $\mathcal{P}_{\mathrm{fin}, 1}(H)$ is $\mathrm{HmF}$ if and only if $H$ is trivial or a cyclic group of order 3 .

Proof. The "if" part is an easy consequence of Theorem 4.13 and Propositions 4.12(i) and 4.7(i), when considering that, if $H$ is trivial or a cyclic group of order 3 , then $1_{H} \neq x^{2} \neq x$ for all $x \in H \backslash\left\{1_{H}\right\}$ and every non-empty subset of $H$ has at most 3 elements.

As for the other direction, suppose $\mathcal{P}_{\text {fin, } 1}(H)$ is $\mathrm{HmF}$ and $H$ is non-trivial. Then $\mathcal{P}_{\text {fin, }, 1}(H)$ is atomic, and we claim that $H$ is a 3 -group. By Theorem 3.9 and Lemma 3.8(ii), it suffices to show that $x^{3} \in\left\{1_{H}, x, x^{2}\right\}$ for every $x \in H$, since this in turn implies (by induction) that $\langle x\rangle_{H} \subseteq\left\{1_{H}, x, x^{2}\right\}$ and $\operatorname{ord}_{H}(x) \leq 3$.

Assume to the contrary that $x^{3} \notin\left\{1_{H}, x, x^{3}\right\}$ for some $x \in H$, and set $X:=\left\{1_{H}, x, x^{2}, x^{3}\right\}$. By Theorem 4.13, $\mathfrak{a}:=\left\{1_{H}, x\right\}^{* 3}$ and $\mathfrak{b}:=\left\{1_{H}, x\right\} *\left\{1_{H}, x^{2}\right\}$ are both factorizations of $X$ in $\mathcal{P}_{\text {fin, }, 1}(H)$; and in light of Proposition 4.7(i), $\mathfrak{b}$ is in fact a minimal factorization (of length 2 ). Then $\mathfrak{a}$ cannot be minimal, because $\mathcal{P}_{\text {fin }, 1}(H)$ is $\mathrm{HmF}$ and $\mathfrak{a}$ has length 3 . However, since $\mathcal{P}_{\text {fin, }, 1}(H)$ is a reduced monoid (and $X$ is not an atom), this is only possible if $x^{3} \in X=\left\{1_{H}, x\right\}^{2}$, a contradiction.

So, $H$ is a 3-group, and as such it has a non-trivial center $Z(H)$, see e.g. [29, Theorem 2.11(i)]. Let $z$ be an element in $Z(H) \backslash\left\{1_{H}\right\}$, and suppose for a contradiction that $H$ is not cyclic. Then we can choose 
some element $y \in H \backslash\langle z\rangle_{H}$, and it follows from the above that $K:=\langle y, z\rangle_{H}$ is an abelian subgroup of $H$ with $\operatorname{ord}_{H}(y)=\operatorname{ord}_{H}(z)=3$ and $|K|=9$. We will prove that $K$ has $\preceq_{\mathcal{P}_{\mathrm{fin}, 1}(H)}$-minimal factorizations of more than one length, which is a contradiction and finishes the proof.

Indeed, we are guaranteed by Theorem 4.13 that $\mathfrak{c}:=\left\{1_{H}, y\right\}^{* 2} *\left\{1_{H}, z\right\}^{* 2}$ is a length- 4 factorization of $K$ in $\mathcal{P}_{\text {fin, }, 1}(H)$; and it is actually a minimal factorization, because removing one or more atoms from c yields an $\mathscr{A}\left(\mathcal{P}_{\text {fin }, 1}(H)\right.$ )-word whose image under $\pi_{\mathcal{P}_{\text {fin }, 1}(H)}$ has cardinality at most 8 (whereas we have already noted that $|K|=9)$. On the other hand, it is not difficult to check that $A:=\left\{1_{H}, y, z\right\}$ is an atom of $\mathcal{P}_{\text {fin }, 1}(H)$ : If $\left\{1_{H}, y, z\right\}=Y Z$ for some $Y, Z \in \mathcal{P}_{\text {fin }, 1}(H)$ with $|Y|,|Z| \geq 2$, then $Y, Z \subseteq\left\{1_{H}, y, z\right\}$ and $Y \cap Z=\left\{1_{H}\right\}$, whence $Y Z=\left\{1_{H}, y\right\} \cdot\left\{1_{H}, z\right\}=K \neq A$. This in turn implies that $A^{* 2}$ is a length-2 factorization of $K$ in $\mathcal{P}_{\mathrm{fin}, 1}(H)$, and it is minimal by Proposition 4.7(i). So, we are done.

Corollary 4.15. Let $H$ be a monoid. Then $\mathcal{P}_{\text {fin }, 1}(H)$ is minimally factorial if and only if $H$ is trivial.

Proof. The "if" part is obvious. For the other direction, assume by way of contradiction that $\mathcal{P}_{\text {fin }, 1}(H)$ is minimally factorial but $H$ is non-trivial. Then $\mathcal{P}_{\text {fin }, 1}(H)$ is $\mathrm{HmF}$, and we obtain from Theorem 4.14 that $H$ is a cyclic group of order 3. Accordingly, let $x$ be a generator of $H$. By Lemma 3.7(i) and Proposition $4.7(\mathrm{i}), \mathfrak{a}:=\left\{1_{H}, x\right\}^{* 2}$ and $\mathfrak{b}:=\left\{1_{H}, x^{2}\right\}^{* 2}$ are both minimal factorizations of $H$ in $\mathcal{P}_{\text {fin }, 1}(H)$. However, $(\mathfrak{a}, \mathfrak{b}) \notin \mathscr{C}_{\mathcal{P}_{\text {fin }, 1}}(H)$, because $\mathcal{P}_{\text {fin }, 1}(H)$ is a reduced monoid. Therefore, $\mathcal{P}_{\text {fin }, 1}(H)$ is not minimally factorial, so leading to a contradiction and completing the proof.

At this point, we have completely characterized the correlation between the ground monoid $H$ and whether $\mathcal{P}_{\text {fin, },}(H)$ has factorization properties such as atomicity, BFness, etc., and their minimal counterparts. In most cases, this extends to a characterization of whether the same properties hold for $\mathcal{P}_{\text {fin, } \times}(H)$, with the exception of the gap suggested by Theorem 4.14 and Corollary 4.15. In particular, it still remains to determine the monoids $H$ which make $\mathcal{P}_{\text {fin }, \times}(H) \mathrm{HmF}$ or minimally factorial. However, what we have shown indicates, we believe, that the arithmetic of $\mathcal{P}_{\text {fin }, 1}(H)$ and $\mathcal{P}_{\text {fin }, \times}(H)$ is robust and ripe for more focused study.

\section{CyClic monoids AND interval Length SETS}

For those monoids $H$ with $\mathcal{P}_{\text {fin, }, 1}(H)$ atomic, we have by Proposition 3.8 that the semigroup generated by an element $x \in H$ is isomorphic either to $\mathbf{Z} / n \mathbf{Z}$ or to $\mathbf{N}$ under addition. As such, we will concentrate throughout on factorizations in $\mathcal{P}_{\text {fin }, 0}(\mathbf{Z} / n \mathbf{Z})$ and also mention some results on $\mathcal{P}_{\text {fin }, 0}(\mathbf{N})$ which are discussed in detail in $[20, \S 4]$. At the end we will return to the general case, where the preceding discussion will culminate in a realization result (Theorem 5.7) for sets of minimal lengths of $\mathcal{P}_{\text {fin }, 1}(H)$.

We invite the reader to review $\S 2.1$ before reading further. Also, note that, through the whole section, we have replaced the notation $\mathcal{P}_{\text {fin }, 1}(H)$ with $\mathcal{P}_{\text {fin }, 0}(H)$ when $H$ is written additively (cf. Example 4.11).

Definition 5.1. Let $X \in \mathcal{P}_{\text {fin }, 0}(\mathbf{Z} / n \mathbf{Z})$. We say that a non-empty factorization $\mathfrak{a}=A_{1} * \cdots * A_{\ell} \in \mathcal{Z}(X)$ is a non-reducible factorization (or, shortly, an NR-factorization) if $\max \hat{A}_{1}+\cdots+\max \hat{A}_{\ell}=\max \hat{X}$.

This condition on factorizations will allow us to bring calculations up to the integers, where sumsets are more easily understood. More importantly, NR-factorizations are very immediately relevant to our investigation of minimal factorizations.

Lemma 5.2. Any NR-factorization in $\mathcal{P}_{\mathrm{fin}, 0}(\mathbf{Z} / n \mathbf{Z})$ is a minimal factorization. 
Proof. Let $\mathfrak{a}=A_{1} * \cdots * A_{\ell}$ be an NR-factorization in $\mathcal{P}_{\text {fin }, 0}(\mathbf{Z} / n \mathbf{Z})$ of length $\ell$, and assume for the sake of contradiction that $\mathfrak{a}$ is not minimal. Since $\mathcal{P}_{\text {fin }, 0}(\mathbf{Z} / n \mathbf{Z})$ is reduced and commutative, the factorizations

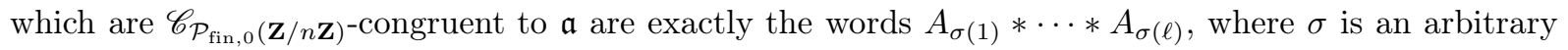
permutation of the interval $\llbracket 1, \ell \rrbracket$. So, on account of Proposition 4.7(i), the non-minimality of $\mathfrak{a}$ implies without loss of generality that $\ell \geq 3$ and $X:=A_{1}+\cdots+A_{\ell}=A_{1}+\cdots+A_{k}$ for some $k \in \llbracket 1, \ell-1 \rrbracket$.

Now, let $x \in X$ such that $\hat{x}=\max \hat{X}$. Using that $\mathfrak{a}$ is an NR-factorization, and considering that, for each $i \in \llbracket 1, \ell \rrbracket, A_{i}$ is an atom of $\mathcal{P}_{\text {fin, }, 0}(\mathbf{Z} / n \mathbf{Z})$ and hence $\max \hat{A}_{i} \geq 1$, it follows from the above that

$$
\hat{x}=\max \hat{A}_{1}+\max \hat{A}_{2}+\cdots+\max \hat{A}_{\ell}>\max \hat{A}_{1}+\cdots+\max \hat{A}_{k},
$$

On the other hand, since $X=A_{1}+\cdots+A_{k}$, there are $a_{1} \in A_{1}, \ldots, a_{k} \in A_{k}$ such that $a_{1}+\cdots+a_{k}=x$, from which we see that $\hat{x} \equiv \hat{a}_{1}+\cdots+\hat{a}_{k} \bmod n$. But it follows from (5) that $0 \leq \hat{a}_{1}+\cdots+\hat{a}_{k}<\hat{x}<n$, and this implies $\hat{x} \not \equiv \hat{a}_{1}+\cdots+\hat{a}_{k} \bmod n$ (recall that, by definition, $\left.\hat{X} \subseteq \llbracket 0, n-1 \rrbracket\right)$. So we got a contradiction, showing that $\mathfrak{a}$ was minimal and completing the proof.

We are aiming to find, for every $k \in \llbracket 2, n-1 \rrbracket$, a set $X_{k} \in \mathcal{P}_{\text {fin }, 0}(\mathbf{Z} / n \mathbf{Z})$ for which $\mathrm{L}^{\mathrm{m}}\left(X_{k}\right)=\llbracket 2, k \rrbracket$, on the assumption that $n \geq 5$ is odd: Most of the difficulty lies in showing that $2 \in \mathrm{L}^{\mathrm{m}}\left(X_{k}\right)$. To do this, we first need to produce some large atoms.

Proposition 5.3. Let $n \geq 5$ be odd. Then the following sets are atoms of $\mathcal{P}_{\text {fin }, 0}(\mathbf{Z} / n \mathbf{Z})$ :

(i) $B_{h}:=\{\overline{0}\} \cup\{\overline{1}, \overline{3}, \ldots, \bar{h}\}$ for odd $h \in \llbracket 1,(n-1) / 2 \rrbracket$.

(ii) $C_{1}:=\{\overline{0}, \overline{2}\}, C_{3}:=\{\overline{0}, \overline{2}, \overline{3}, \overline{4}\}$, and $C_{\ell}:=B_{\ell} \cup\{\overline{\ell+1}\}$ for odd $\ell \in \llbracket 5,(n-1) / 2 \rrbracket$.

Proof. (i) Let $h \in \llbracket 1,(n-1) / 2 \rrbracket$ be odd, and suppose that $B_{h}=X+Y$ for some $X, Y \in \mathcal{P}_{\text {fin }, 0}(\mathbf{Z} / n \mathbf{Z})$. Then $X$ and $Y$ are subsets of $B_{h}$, so

$$
\max \hat{X}+\max \hat{Y} \leq 2 \max \hat{B}_{h}=2 h \leq n-1 \text {. }
$$

Because $\overline{1} \in B_{h}$, we must have $\overline{1} \in X \cup Y$. However, if $\overline{1} \in X$ and $a \in Y$ for some $a \in B_{h} \backslash\{\overline{0}\}$, then $1+\hat{a} \in \hat{X}+\hat{Y}$ is even, which is impossible since $\max \hat{X}+\max \hat{Y}<n$ and $\hat{B}_{h} \backslash\{0\}$ consists only of odd numbers. Thus $Y=\{\overline{0}\}$, and hence $B_{h}$ is an atom.

(ii) $C_{1}$ is an atom by Lemma $3.7(\mathrm{i})$ and it is not too difficult to see that so is $C_{3}$. Therefore, let $\ell \geq 5$ and suppose $C_{\ell}=X+Y$ for some $X, Y \in \mathcal{P}_{\text {fin, } 0}(\mathbf{Z} / n \mathbf{Z})$ with $X, Y \neq\{\overline{0}\}$.

First assume that $\overline{\ell+1} \notin X \cup Y$. Then $\hat{X}$ and $\hat{Y}$ consist only of odd integers, so $\hat{x}+\hat{y}$ is an even integer in the interval $\llbracket 2, n-1 \rrbracket$ for all $x \in X \backslash\{\overline{0}\}$ and $y \in Y \backslash\{\overline{0}\}$. However, $\hat{X}+\hat{Y}=\hat{C}_{\ell}$ and the only non-zero even element of $\hat{C}_{\ell}$ is $\ell+1$. Thus, it must be that $X=\{\overline{0}, x\}$ and $Y=\{\overline{0}, y\}$ for some non-zero $x, y \in \mathbf{Z} / n \mathbf{Z}$, with the result that $|X+Y| \leq 4<\left|C_{\ell}\right|$, a contradiction.

It follows (without loss of generality) that $\overline{\ell+1} \in Y$. Then $X \subseteq\{\overline{0}, \bar{\ell}, \overline{\ell+1}\}$, for, if $x \in X$ with $0<\hat{x}<\ell$, then $\hat{x}+\ell+1 \in \hat{C}_{\ell}$, which is impossible since $\hat{x}+\ell+1 \in \llbracket \max \hat{C}_{\ell}+1, n-1 \rrbracket$. This in turn implies that $Y \subseteq\{\overline{0}, \overline{1}, \bar{\ell}, \overline{\ell+1}\}$ for similar reasons. As a consequence,

$$
X+Y \subseteq\{\overline{0}, \bar{\ell}, \overline{\ell+1}\}+\{\overline{0}, \overline{1}, \bar{\ell}, \overline{\ell+1}\}=\{\overline{0}, \overline{1}, \bar{\ell}, \overline{\ell+1}, \overline{2 \ell}, \overline{2 \ell+1}, \overline{2 \ell+2}\}
$$

However, $\ell+1<2 \ell \leq n-1$, so we cannot have $\overline{2 \ell} \in X+Y$. Then $2 \ell+1=n$, in which case $\overline{2 \ell+1}=\overline{0}$ and $\overline{2 \ell+2}=\overline{1}$; or $2 \ell+1<n$, so that $\overline{2 \ell+1}, \overline{2 \ell+2} \notin C_{h}$ (recall that $\ell \leq(n-1) / 2$ ). In either case, we get $X+Y \subseteq\{\overline{0}, \overline{1}, \bar{\ell}, \overline{\ell+1}\}$, hence $|X+Y| \leq 4<\left|C_{\ell}\right|$, which is a contradiction and leads us to conclude that $C_{\ell}$ is an atom. 
Now that we have found large atoms in $\mathcal{P}_{\text {fin, } 0}(\mathbf{Z} / n \mathbf{Z})$, we can explicitly give, for each $k \in \llbracket 2, n-1 \rrbracket$, an element $X_{k} \in \mathcal{P}_{\text {fin }, 0}(\mathbf{Z} / n \mathbf{Z})$ which has a (minimal) factorization of length 2 .

Lemma 5.4. Fix an odd integer $n \geq 5$ and let $k \in \llbracket 2, n-1 \rrbracket$. Then the set $X_{k}=\{\overline{0}, \overline{1}, \ldots, \bar{k}\}$ has an NR-factorization into two atoms in $\mathcal{P}_{\text {fin }, 0}(\mathbf{Z} / n \mathbf{Z})$.

Proof. We will use the atoms $B_{h}$ and $C_{\ell}$ as defined in Proposition 5.3. We claim that, for every $r \in\{0,1\}$ and all odd $h \in \llbracket 1,(n-1) / 2 \rrbracket$,

$$
\hat{B}_{h+2 r}+\hat{C}_{h}=\llbracket 0,2 h+2 r+1 \rrbracket \quad \text { and } \quad \hat{C}_{h+2 r}+\hat{C}_{h}=\llbracket 0,2 r+2 h+2 \rrbracket .
$$

We will only demonstrate that $\hat{B}_{h}+\hat{C}_{h}=\llbracket 0,2 h+1 \rrbracket($ the other cases are an easy consequence). The claim is trivial if $h=1$ or $h=3$, so suppose $h \geq 5$. Then

$$
\hat{B}_{h}+\hat{C}_{h} \supseteq\{1,3, \ldots, h\}+\{0, h+1\}=\{1,3, \ldots, 2 h+1\}
$$

and

$$
\hat{B}_{h}+\hat{C}_{h} \supseteq\{1,3, \ldots, h\}+\{1, h\}=\{2,4, \ldots, 2 h\},
$$

so $\hat{B}_{h}+\hat{C}_{h} \supseteq \llbracket 0,2 h+1 \rrbracket$. This gives that $\hat{B}_{h}+\hat{C}_{h}=\llbracket 0,2 h+1 \rrbracket$, since $\max \hat{B}_{h}+\max \hat{C}_{h}=h+(h+1)$.

Accordingly, we now prove that $X_{k}$ can be expressed as a two-term sum involving $B_{h}$ and $C_{\ell}$, for some suitable choices of $h$ and $\ell$ depending on the parity of $k$.

CASE 1: $k=2 m+1$ (i.e., $k$ is odd). Then it is immediate to verify that $X_{k}=B_{m}+C_{m}$ if $m$ is odd, and $X_{k}=B_{m+1}+C_{m-1}$ if $m$ is even.

CAse 2: $k=2 m$ (i.e., $k$ is even). Since $X_{2}=B_{1}+B_{1}$ and $X_{4}=B_{1}+B_{3}$, we may assume $m \geq 3$. Then it is seen that $X_{k}=C_{m}+C_{m-2}$ if $m$ is odd, and $X_{k}=C_{m-1}+C_{m-1}$ if $m$ is even.

We are left to show that the decompositions given above do in fact correspond to minimal factorizations. As an example, consider the case when $k=2 m+1$ and $m$ is odd (the computation will be essentially identical in the other cases). Then $\max \hat{B}_{m}+\max \hat{C}_{m}=2 m+1$, so that $B_{m} * C_{m}$ is an NR-factorization of $X_{k}$, and is hence minimal by Proposition 5.2 .

Lemma 5.5. Fix an odd integer $n \geq 3$ and, for each $k \in \llbracket 2, n-1 \rrbracket$, let $X_{k}:=\{\overline{0}, \overline{1}, \ldots, \bar{k}\} \in \mathcal{P}_{\text {fin }, 0}(\mathbf{Z} / n \mathbf{Z})$. Then $\mathrm{L}^{\mathrm{m}}\left(X_{k}\right)=\llbracket 2, k \rrbracket$.

Proof. We have already established in Lemma 5.4 that $X_{2}$ has an NR-factorization of length 2. Now fix $k \in \llbracket 3, n-1 \rrbracket$ and suppose that, for all $h \in \llbracket 2, k-1 \rrbracket$ and $\ell \in \llbracket 2, h \rrbracket, X_{h}$ has an NR-factorization of length $\ell$. Choose some $\ell \in \llbracket 2, k-1 \rrbracket ; X_{k-1}$ has an NR-factorization $\mathfrak{a}$, and it is straightforward to see that $\{\overline{0}, \overline{1}\} * \mathfrak{a}$ is an NR-factorization of $X_{k}$. Letting $\ell$ range over $\llbracket 2, k-1 \rrbracket$, this argument, Lemma 5.2, and Lemma 5.4 imply that $\mathrm{L}^{\mathrm{m}}\left(X_{k}\right) \supseteq \llbracket 2, k \rrbracket$. Moreover, Proposition 4.12(i) yields the other inclusion and so we have $\mathrm{L}^{\mathrm{m}}\left(X_{k}\right)=\llbracket 2, k \rrbracket$.

Lemma 5.6. Let $H$ be a non-torsion monoid. Then $\mathscr{L}\left(\mathcal{P}_{\text {fin }, 0}(\mathbf{N})\right) \subseteq \mathscr{L}^{\mathrm{m}}\left(\mathcal{P}_{\text {fin }, 1}(H)\right)$, and for every $k \geq 2$ there exists $Y_{k} \in \mathcal{P}_{\text {fin, } 1}(H)$ with $\mathrm{L}^{\mathrm{m}}\left(Y_{k}\right)=\llbracket 2, k \rrbracket$.

Proof. Suppose that $y \in H$ has infinite order, and set $Y:=\left\{y^{k}: k \in \mathbf{N}\right\}$. Clearly, $Y$ is a submonoid of $H$, and the (monoid) homomorphism $(\mathbf{N},+) \rightarrow Y: k \mapsto y^{k}$ determined by sending 1 to $y$ induces an 
isomorphism $\mathcal{P}_{\text {fin }, 0}(\mathbf{N}) \rightarrow \mathcal{P}_{\text {fin }, 1}(Y)$. Since, by Proposition 3.2(iii), $\mathcal{P}_{\text {fin }, 1}(Y)$ is a divisor-closed submonoid of $\mathcal{P}_{\text {fin }, 1}(H)$, we thus have by parts (iv) and (v) of Proposition 4.7 that

$$
\mathscr{L}\left(\mathcal{P}_{\text {fin }, 0}(\mathbf{N})\right)=\mathscr{L}^{\mathrm{m}}\left(\mathcal{P}_{\text {fin }, 0}(\mathbf{N})\right)=\mathscr{L}^{\mathrm{m}}\left(\mathcal{P}_{\text {fin }, 1}(Y)\right) \subseteq \mathscr{L}^{\mathrm{m}}\left(\mathcal{P}_{\text {fin }, 1}(H)\right)
$$

The rest of the statement now follows from the above and [20, Proposition 4.8].

Theorem 5.7. Assume $H$ is a monoid such that $1_{H} \neq x^{2} \neq x$ for all $x \in H \backslash\left\{1_{H}\right\}$, and set $N:=$ $\sup \left\{\operatorname{ord}_{H}(x): x \in H\right\}$. Then $\llbracket 2, k \rrbracket \in \mathscr{L}^{\mathrm{m}}\left(\mathcal{P}_{\text {fin, }, 1}(H)\right)$ for every $k \in \llbracket 2, N-1 \rrbracket$.

Proof. If $H$ is non-torsion, this follows immediately from Lemma 5.6. Otherwise, let $k \in \llbracket 2, N-1 \rrbracket$ and $y \in H$ with $n:=\operatorname{ord}_{H}(x)>k$. Then $Y:=\langle y\rangle_{H} \cong \mathbf{Z} / n \mathbf{Z}$, so we have by Proposition 3.2(iii), Lemma 5.5, and Proposition $4.7($ iv $)$ that $\llbracket 2, k \rrbracket \in \mathscr{L}^{\mathrm{m}}\left(\mathcal{P}_{\text {fin }, 1}(Y)\right) \subseteq \mathscr{L}^{\mathrm{m}}\left(\mathcal{P}_{\text {fin }, 1}(H)\right)$.

\section{Closing Remarks}

This preliminary foray into minimal factorizations raises several questions. In particular, to what extent can finite subsets of $\mathbf{N}_{\geq 2}$ be realized as sets of minimal lengths of some power monoid? Which families of subsets can be simultaneously realized by a single power monoid?

There are also questions beyond sets of lengths that can be addressed; we should expect to be able to formulate and study the "minimal versions" of other invariants commonly considered in Factorization Theory (unions of length sets, sets of distances, elasticities, catenary and tame degrees, etc.). The results we have seen in the present paper suggest that the study of these types of invariants in power monoids is almost never trivial.

\section{ACKNOWLEDGMENTS}

We are grateful to Qinghai Zhong for helping with the proof of Theorem 3.11(i); to Alfred Geroldinger for several productive discussions; and to an anonymous reviewer for comments that pushed us to improve this manuscript. The first-named author also wishes to thank the OSU Department of Mathematics for the opportunity to travel for an extended period, and everyone at the Institute for Mathematics and Scientific Computing at University of Graz for being gracious hosts.

\section{REFERENCES}

[1] A. G. Aḡargün, D. D. Anderson, and S. Valdes-Leon, Factorization in commutative rings with zero divisors, III, Rocky Mountain J. Math. 31 (2001), No. 1, 1-21.

[2] D. D. Anderson (ed.), Factorization in Integral Domains, Lect. Notes Pure Appl. Math. 189, Marcel Dekker, 1997.

[3] D. D. Anderson and R. Markanda, Unique Factorization Rings With Zero Divisors, Houston J. Math. 11 (1985), No. 3, 423-426.

[4] D. D. Anderson and R. Markanda, Unique Factorization Rings With Zero Divisors: Corrigendum, Houston J. Math. 11 (1985), No. 1, 15-30.

[5] D. D. Anderson and S. Valdes-Leon, Factorization in Commutative Rings with Zero Divisors, Rocky Mountain J. Math. 26 (1996), No. 2, 439-480.

[6] D. D. Anderson and S. Valdes-Leon, "Factorization in commutative rings with zero divisors, II", pp. 197-219 in [2].

[7] M. Axtell, S. Forman, N. Roersma, and J. Stickles, Properties of U-factorizations, Int. J. Commut. Rings 2 (2003), No. 2, 83-99.

[8] N. R. Baeth, B. Burns, and J. Mixco, A fundamental theorem of modular arithmetic, Period. Math. Hungar. 75 (2017), No. 2, 356-367. 
[9] N. R. Baeth and A. Geroldinger. Monoids of modules and arithmetic of direct-sum decompositions, Pacific J. Math. 271 (2014), 257-319.

[10] N. R. Baeth and D. Smertnig, Factorization theory: From commutative to noncommutative settings, J. Algebra 441 (2015), 475-551.

[11] N. R. Baeth and R. Wiegand, Factorization Theory and Decompositions of Modules, Amer. Math. Monthly 120 (2013), No. 1, 3-34.

[12] P. Baginski and S. T. Chapman, Factorizations of algebraic integers, block monoids, and additive number theory, Amer. Math. Monthly 118 (2011), No. 10, 901-920.

[13] A. Bouvier, Anneaux présimplifiables, Rev. Roumaine Math. Pures Appl. 19 (1974), 713-724.

[14] A. Bouvier, Structure des anneaux á factorisation unique, Pub. Dépt. Math. Lyon 11 (1974), No. 3, 39-49.

[15] S. T. Chapman (ed.), Arithmetical Properties of Commutative Rings and Monoids, Lect. Notes Pure Appl. Math. 241, Chapman \& Hall/CRC, 2005.

[16] S. Chapman, M. Fontana, A. Geroldinger, and B. Olberding (eds.), Multiplicative Ideal Theory and Factorization Theory: Commutative and Non-Commutative Perspectives, Springer Proc. Math. Stat. 170, Springer, 2016.

[17] S. T. Chapman and S. Glaz (eds.), Non-Noetherian Commutative Ring Theory, Math. Appl. 520, Springer, 2000.

[18] S. Chun and D. D. Anderson, Irreducible elements in commutative rings with zero divisors, II, Houston J. Math. 39 (2013), No. 3, 741-752.

[19] S. Chun, D. D. Anderson, and S. Valdes-Leon, Reduced factorizations in commutative rings, Comm. Algebra 39 (2011), No. 5, 1583-1594.

[20] Y. Fan and S. Tringali, Power monoids: A bridge between Factorization Theory and Arithmetic Combinatorics, J. Algebra 512 (Oct 2018), 252-294.

[21] C. R. Fletcher, Unique Factorization Rings, Proc. Camb. Phil. Soc. 65 (1969), 579-583.

[22] M. Fontana, E. Houston, and T. Lucas, Factoring Ideals in Integral Domains, Lect. Notes Unione Mat. Ital. 14, Springer, 2013.

[23] S. Galovich, Unique factorization rings with zero divisors, Math. Mag. 51 (1978), No. 5, 276-283.

[24] A. Geroldinger, Sets of lengths, Amer. Math. Monthly 123 (2016), No. 10, 960-988.

[25] A. Geroldinger and F. Halter-Koch, Non-Unique Factorizations. Algebraic, Combinatorial and Analytic Theory, Pure Appl. Math. 278, Chapman \& Hall/CRC, 2006.

[26] A. Geroldinger and G. Lettl, Factorization problems in semigroups, Semigroup Forum 40 (1990), No. 1, 23-38.

[27] A. Geroldinger and I. Z. Ruzsa, Combinatorial Number Theory and Additive Group Theory, Adv. Courses Math. CRM Barcelona, Birkhäuser, 2009.

[28] A. Geroldinger and Q. Zhong, Factorization theory in commutative monoids, Semigroup Forum 100 (2020), $22-51$.

[29] D. Gorenstein, Finite Groups, Chelsea Pub. Co., 1980 (2nd ed.).

[30] D. J. Grynkiewicz, Structural Additive Theory, Dev. Math. 30, Springer, 2013

[31] M. B. Nathanson, Additive Number Theory: Inverse Problems and the Geometry of Sumsets, Grad. Texts in Math. 165, Springer, 1996.

[32] D. Smertnig, Factorizations in Bounded Hereditary Noetherian Prime Rings, Proc. Edinb. Math. Soc. 62 (2019), No. 2, 395-442.

[33] T. Tao and V.H. Vu, Additive Combinatorics, Cambridge Stud. Adv. Math. 105, Cambridge Univ. Press, 2006.

[34] S. Tringali, Structural properties of subadditive families with applications to factorization theory, Israel J. Math. 234 (2019), No. 1, 1-35.

[35] T. A. Whitelaw, Introduction to abstract algebra, Blackie, 1988 (2nd ed.). 
Department of Mathematics, The Ohio State University | 231 W. 18th Avenue, Columbus, OH 43202, USA

Email address: antoniou.6@osu.edu

URL: https://people.math.osu.edu/antoniou.6/

Institute for Mathematics and Scientific Computing, University of Graz, NAWi Graz | Heinrichstr. 36 , 8010 Graz, Austria

Current address: School of Mathematical Sciences, Hebei Normal University | No. 20 Road East, 2nd Southern Ring, Yuhua District | Shijiazhuang, Hebei, 050024 China

Email address: salvo.tringali@gmail.com

$U R L$ : https://imsc.uni-graz.at/tringali 